\title{
Infinite canonical super-Brownian motion and scaling limits
}

\author{
Remco van der Hofstad *
}

July 15, 2021

\begin{abstract}
We construct a measure valued Markov process which we call infinite canonical superBrownian motion, and which corresponds to the canonical measure of super-Brownian motion conditioned on non-extinction. Infinite canonical super-Brownian motion is a natural candidate for the scaling limit of various random branching objects on $\mathbb{Z}^{d}$ when these objects are (a) critical; (b) mean-field and (c) infinite. We prove that ICSBM is the scaling limit of the spread-out oriented percolation incipient infinite cluster above 4 dimensions and of incipient infinite branching random walk in any dimension. We conjecture that it also arises as the scaling limit in various other models above the upper-critical dimension, such as the incipient infinite lattice tree above 8 dimensions, the incipient infinite cluster for unoriented percolation, uniform spanning trees above 4 dimensions, and invasion percolation above 6 dimensions. This paper also serves as a survey of recent results linking super-Brownian to scaling limits in statistical mechanics.
\end{abstract}

\section{Introduction}

Over the past years, it has become clear that super-Brownian motion (SBM) arises as the scaling limit in various critical systems. Convergence towards SBM can be expected when (a) the system is critical; (b) the particles in the system are moving, and undergo (critical) branching; and (c) the interaction in the system is weak. In practice, requirement (c) means that the system should be above the upper critical dimension, where the scaling ceases to depend on the dimension. Therefore, a Gaussian limit can be expected, and this Gaussian limit is super-Brownian motion. Examples where such results have been (partially) proved are lattice trees above 8 dimensions [15, 16, 40, the voter model above 2 dimensions [10, 11, the contact process above 4 dimensions [17, 37], oriented percolation above 4 dimensions [39] and percolation above 6 dimensions [29, 30]. Often, though not always, the proof of the convergence to SBM uses the lace expansion.

Super-Brownian motion is the principle example of a measure-valued Markov process in a similar way as Brownian motion is the principle example of a diffusion. SBM has attracted considerable attention in the probability literature, and has been described in detail in several recent books and major reviews [12, 19, 21, 150, 57. The canonical measure of super-Brownian motion is described in [50, 57], and describes the structure of a single continuum tree embedded into $\mathbb{R}^{d}$ where particles undergo critical branching at any time scale, and move according to Brownian

*Department of Mathematics and Computer Science, Eindhoven University of Technology, P.O. Box 513, 5600 MB Eindhoven, The Netherlands. E-mail rhofstad@win.tue.nl 
motions. See also 63] for an excellent nontechnical introduction describing scaling limits and their relations to super-processes.

There are two versions of convergence of a single geometric object towards super-Brownian motion. In the first, the size of the critical object, for example the critical percolation cluster, is fixed to be equal to $N$, and the scaling limit as $N \rightarrow \infty$ is taken. In this case, the scaling limit is integrated super-Brownian excursion (ISE), which is SBM conditioned to have total mass 1 . In the second, we investigate what happens when these objects do not die out for a long time. In this case, the scaling limit is the canonical measure of super-Brownian motion. We will describe this limit in detail in Section 3 below, as an introduction to super-Brownian motion for the non-specialist.

The canonical measure of super-Brownian motion is the scaling limit of a single critical branching random walk which starts at the origin and survives for some positive rescaled time. This connection will be made precise in Sections 2 and 3 . Since critical branching processes die out almost surely, also the canonical measure for SBM dies out almost surely. In the above-mentioned examples, it is expected that the critical structures live a finite amount of time almost surely, and this illustrates why SBM can serve as the scaling limit for these objects above the upper critical dimension.

Super-Brownian motion, however, cannot describe the scaling limit of critical systems where the structures almost surely live an infinite amount of time. Examples of such systems are invasion percolation and uniform spanning trees. The aim of this paper is to study a critical super-process which survives with probability one, and can be obtained as an appropriate limit of super-Brownian motion. It is natural to expect that this object, which we call infinite canonical super-Brownian motion, serves as the universal scaling limit of systems that are (a) infinite, though critical; (b) undergo branching and motion; and (c) have weak interaction. There are different versions of such infinite structures, namely incipient infinite structures, which are obtained by appropriate limiting procedures in models where the structures are a.s. finite, and infinite structures, where such a limit is not necessary. An example for the former is the incipient infinite percolation cluster, an example for the latter is a single tree in the uniform spanning forest.

This paper contains four main parts. In the first part (Section 2), we define incipient infinite branching random walk, which is branching random walk conditioned on non-extinction. This can be seen as a warm-up problem for the construction of infinite canonical super-Brownian motion (ICSBM) in the second part (Section 31). We also discuss properties of ICSBM there. In the third part (Section 4), we prove that the incipient infinite cluster in oriented percolation above $4+1$ dimensions converges to ICSBM. The latter result is based on the results obtained in [32], which is in turn based upon the convergence of finite-dimensional distributions proved in [39]. In fact, it is fair to say that this paper is inspired by these two papers. Finally, in the fourth part (Sections 566), we conjecture that many other models also scale to ICSBM, making ICSBM a universal object.

This paper has two aims. The first aim is to introduce and investigate infinite canonical super-Brownian motion, to state results concerning convergence towards ICSBM, and to make conjectures in models where we cannot (yet?) prove such convergence. The second aim of this paper is to review super-Brownian motion and the recent results on super-Brownian motion arising as the scaling limit in various critical high-dimensional models. This paper will attempt not to be technical, and is aimed for the non-specialists both in the field of the applications, as well as in the field of super-processes. As a warm-up, we start by defining incipient infinite branching random walk, which is branching random walk conditioned on non-extinction, and we will see that ICSBM serves as the scaling limit of incipient infinite branching random walk in a similar way as super-Brownian motion serves as a scaling limit for ordinary branching random walk. 


\section{Incipient infinite branching random walk}

In this section, we will construct the incipient infinite branching random walk (IIBRW) measure, which is the measure of branching random walk conditioned on non-extinction. Since SBM is the scaling limit of branching random walk, it is instructive to perform the construction first for branching random walk. This construction is simpler due to the discrete nature of branching random walk, and its close connection to branching processes. We can think of IIBRW as an embedding of a critical branching process conditioned on non-extinction into $\mathbb{Z}^{d}$. Branching process conditioned on non-extinction have a long history, which we will review in some detail below.

We have two constructions for IIBRW. We will see that the two definitions of IIBRW coincide. In the next section, we will construct ICSBM in two ways, and these constructions mirror the two constructions for IIBRW given here.

\subsection{Model and main results}

We start by introducing branching random walk. We follow the construction in [8]. Branching random walk is defined in terms of embeddings of abstract trees into $\mathbb{Z}^{d}$. The abstract trees are the family trees of the critical branching process with a critical offspring distribution $\left(p_{m}\right)_{m=0}^{\infty}$ with finite variance. For simplicity, we will assume that $\left(p_{m}\right)_{m=0}^{\infty}$ has a finite third moment.

In more detail, we begin with a single individual having $\xi$ offspring, where $\xi$ is a random variable with distribution $\left(p_{m}\right)_{m=0}^{\infty}$, i.e., $\mathbb{P}(\xi=m)=p_{m}$ with

$$
\sum_{m} m p_{m}=1, \quad \sigma_{p}^{2}=\sum_{m} m(m-1) p_{m}<\infty .
$$

Each of the offspring then independently has offspring of its own, with the same critical distribution $\left(p_{m}\right)_{m=0}^{\infty}$. For a tree $T$, with the $i^{\text {th }}$ individual having $\xi_{i}$ offspring, this associates to $T$ the probability

$$
\mathbb{P}(T)=\prod_{i \in T} p_{\xi_{i}}
$$

The product is over the vertices of $T$.

It is important to be clear about when two trees $T$ are the same and when they are not. For this, we introduce a description of $T$ in terms of words. These words arise inductively as follows. The root is the word 0 . The children of the root are the words $01,02, \ldots, 0 \xi_{0}$. The children of 01 are the words $011, \ldots, 01 \xi_{01}$, and so on. The family tree is then uniquely represented by a set of words. Two trees are the same if and only if they are represented by the same set of words.

We define an embedding $\phi$ of $T$ into $\mathbb{Z}^{d}$ to be a mapping from the vertices of $T$ into $\mathbb{Z}^{d}$ such that the root is mapped to the origin and, given that $i$ is mapped to $x \in \mathbb{Z}^{d}$, the child $j$ of $i$ is mapped to $y \in \mathbb{Z}^{d}$ with probability $D(y-x)$. We will always assume that $D$ is symmetric, and that $D$ has finite variance, i.e.,

$$
\sigma^{2}=\sum_{x \in \mathbb{Z}^{d}}|x|^{2} D(x)<\infty
$$

where $|\cdot|$ denotes the Euclidean norm on $\mathbb{R}^{d}$. We will also assume that for some $\delta \in(0,1)$,

$$
\sigma^{2}=\sum_{x \in \mathbb{Z}^{d}}|x|^{2+2 \delta} D(x)<\infty
$$

In later sections, we will put stronger conditions on $D$, but for branching random walk, this is not necessary. 
Branching random walk is then defined to be the set of configurations $(T, \phi)$, with probabilities

$$
\mathbb{P}^{(\text {brw })}(T, \phi)=\mathbb{P}(T) \prod_{i j \in T} D(\phi(j)-\phi(i)) .
$$

Here $i j \in T$ means that $j$ is the child of $i$ in the tree $T$. In particular, the path in $\mathbb{Z}^{d}$ from the origin to $\phi(i)$, where $i \in T$ is a random walk path of length $|i|$ with transition probabilities given by $D$. Here $|i|$ denotes the generation of $i$ in $T$, which is the same as the graph distance between the root of $T$ and $i$.

Critical branching processes die out, i.e., $\mathbb{P}(|T|<\infty)=1$. We now give two ways of generating a measure on infinite trees. We let $T_{m}$ denote the restriction of $T$ to the points that are at most tree distance $m$ away from the root, i.e., $T_{m}=\{i \in T:|i| \leq m\}$. Then, we let $(T, \phi)_{m}$ denote the embedding of $T_{m}$ in $\mathbb{Z}^{d}$.

Firstly, for a realisation $C$ of the embedded tree up to time $m$, we define

$$
\mathbb{P}_{n}^{\text {(brw) }}(C)=\sum_{x \in \mathbb{Z}^{d}} \sum_{i \in T:|i|=n} \mathbb{P}^{\text {(brw) }}\left((T, \phi)_{m}=C, \phi(i)=x\right),
$$

and we let

$$
\mathbb{P}_{\infty}^{(\text {brw })}(C)=\lim _{n \rightarrow \infty} \mathbb{P}_{n}^{(\text {brw })}(C),
$$

assuming the limit exists. Denote by

$$
N_{n}=\#\{i \in T:|i|=n\}
$$

the number of particles alive at time $n$. Then

$$
\mathbb{P}_{n}^{\text {(brw) }}(C)=\mathbb{E}^{\text {(brw) }}\left(N_{n} I\left[(T, \phi)_{m}=C\right]\right),
$$

where, for an event $E, I[E]$ denotes the indicator of $E$. The measure $\mathbb{P}_{n}^{(\text {brw })}$ is a probability measure, since $\left\{N_{n}\right\}_{n=0}^{\infty}$ is a martingale with $N_{0}=1$. Therefore, $\mathbb{P}_{n}^{\text {(brw) }}$ is a martingale transformation of $\mathbb{P}^{(\text {brw })}$.

For our second construction, we let

$$
\mathbb{Q}_{n}^{\text {(brw) }}(C)=\mathbb{P}^{\text {(brw) }}\left((T, \phi)_{m}=C|\exists i \in T:| i \mid=n\right),
$$

and we let

$$
\mathbb{Q}_{\infty}^{\text {(brw) }}(C)=\lim _{n \rightarrow \infty} \mathbb{Q}_{n}^{\text {(brw) }}(C),
$$

assuming the limit exists. The conditioning that there is an $i \in T$ with $|i|=n$ means that we condition the branching process to be alive at time $n$.

Theorem 2.1. The measures in (2.7) and (2.11) are well-defined and $\mathbb{P}_{\infty}^{(\text {brw })}=\mathbb{Q}_{\infty}^{\text {(brw) }}$.

Proof. The events $(T, \phi)_{m}=C$ are cylinder events, and we will prove convergence for these cylinder events first. When $(T, \phi)_{m}=C$, we have that $N_{m}=N_{m}(C)$, which is the number of particles in generation $m$ for $C$. We will first show

$$
\mathbb{P}_{\infty}^{\text {(brw) }}(C)=N_{m}(C) \mathbb{P}^{\text {(brw })}\left((T, \phi)_{m}=C\right)=\mathbb{P}_{m}^{\text {(brw) }}(C) .
$$

Equation (2.12) shows that $\mathbb{P}_{\infty}^{(\text {brw })}$ can be seen as a martingale change of measure of $\mathbb{P}^{\text {(brw) }}$. Such transformations occur more generally for conditioned stochastic processes, and are often called $h$ transforms. See [61] for an example where the $h$-transform is used to compute the super-Brownian motion exit measure. 
We compute for every $n \geq m$

$$
\begin{aligned}
\mathbb{P}_{n}^{\text {(brw) }}(C) & =\mathbb{E}^{\text {(brw) }}\left(N_{n} I\left[(T, \phi)_{m}=C\right]\right) \\
& =\mathbb{P}^{\text {(brw })}\left((T, \phi)_{m}=C\right) \mathbb{E}^{\text {(brw })}\left(N_{n} \mid(T, \phi)_{m}=C\right) \\
& =\mathbb{P}^{\text {(brw })}\left((T, \phi)_{m}=C\right) \mathbb{E}^{\text {(brw })}\left(N_{n} \mid N_{m}=N_{m}(C)\right)=N_{m}(C) \mathbb{P}^{(\text {brw })}\left((T, \phi)_{m}=C\right)
\end{aligned}
$$

since $\left\{N_{m}\right\}_{m=0}^{\infty}$ is a martingale. As the right-hand side does not depend on $n$, we also must have that (2.12) holds.

We will next show that also $\mathbb{Q}_{\infty}^{(\text {brw })}(C)$ equals the right-hand side of $(2.12)$, which will prove Theorem 2.1. We first let

$$
\theta_{n}=\mathbb{P}^{\text {(brw) }}(\exists i \in T:|i|=n) .
$$

We again compute, for $n \geq m$, and using that $\left(N_{n}\right)_{n=0}^{\infty}$ is a martingale,

$$
\begin{aligned}
\mathbb{Q}_{n}^{\text {(brw) }}(C) & =\frac{1}{\theta_{n}} \mathbb{P}^{\text {(brw })}\left((T, \phi)_{m}=C, \exists i \in T:|i|=n\right) \\
& =\frac{1}{\theta_{n}} \mathbb{P}^{\text {(brw })}\left((T, \phi)_{m}=C\right) \mathbb{P}^{\text {(brw })}\left(\exists i \in T:|i|=n \mid(T, \phi)_{m}=C\right) \\
& =\frac{1}{\theta_{n}} \mathbb{P}^{\text {(brw })}\left((T, \phi)_{m}=C\right) \mathbb{P}^{\text {(brw })}\left(\exists i \in T:|i|=n \mid N_{m}=N_{m}(C)\right) \\
& =\mathbb{P}^{\text {(brw })}\left((T, \phi)_{m}=C\right) \frac{1-\left(1-\theta_{n-m}\right)^{N_{m}(C)}}{\theta_{n}},
\end{aligned}
$$

where in the final equality, we use that the particles in the first generation evolve independently. As $n \rightarrow \infty$, the right-hand side of (2.15) converges to $N_{m}(C) \mathbb{P}^{\text {(brw })}\left((T, \phi)_{m}=C\right)$ whenever $\theta_{n} \rightarrow 0$, and $\frac{\theta_{n}}{\theta_{n-m}} \rightarrow 1$. In fact, for branching random walk, we know a lot more (see e.g. [2]), namely that

$$
\lim _{n \rightarrow \infty} n \theta_{n}=\frac{2}{\sigma_{p}^{2}} .
$$

We now complete the proof of Theorem 2.1. Cylinder events of the form $(T, \phi)_{m}=C$ generate the $\sigma$-algebra of all events. Since the limiting measure $\mathbb{P}_{\infty}^{(\text {brw })}$ is consistent, we can extend it to the full $\sigma$-algebra by Kolmogorov's Extension Theorem (see e.g. [62]). This completes the proof.

\subsection{The branching random walk higher-point functions}

A convenient way to describe the distribution of a discrete random measure is by using the $r$-point functions. In this section, we will assume that $\left(p_{m}\right)_{m=0}^{\infty}$ has all moments. The $r$-point function describe the numbers and locations of particles present at various times. Denote by

$$
\tau_{n_{1}, \ldots, n_{r-1}}\left(x_{1}, \ldots, x_{r-1}\right)=\sum_{i_{1}, \ldots, i_{r-1}} \mathbb{P}^{\text {(brw })}\left(i_{j} \in T, \phi\left(i_{j}\right)=x_{j},\left|i_{j}\right|=n_{j} \text { for each } j=1, \ldots, r-1\right)
$$

the branching random walk $r$-point functions. We will often abbreviate

$$
\tau_{\vec{n}}(\vec{x})=\tau_{n_{1}, \ldots, n_{r-1}}\left(x_{1}, \ldots, x_{r-1}\right) .
$$

The $r$-point functions give rise to measures, and these measure are called mean moment measures, or just moment measures. Indeed, let $n \geq 0$, and define the random measures $\left\{\mu_{n}\right\}_{n=0}^{\infty}$ by

$$
\mu_{n}(x)=\sum_{i \in T:|i|=n} I[\phi(i)=x] .
$$


The random measures $\left\{\mu_{n}\right\}_{n=0}^{\infty}$ evolve in time and $\mu_{n}$ describes the amount of mass and the spatial location of the mass of the BRW at time $n$. We start with a single particle at time 0 located at the origin, so that

$$
\mu_{0}(x)=\delta_{x, 0} .
$$

Then, the law of the measured-valued process $\left\{\mu_{n}\right\}_{n=0}^{\infty}$ can be computed in terms of the joint moments $\mathbb{E}^{\text {(brw) }}\left[\prod_{i=1}^{r-1} \mu_{m_{i}}\left(y_{i}\right)^{a_{i}}\right]$, where $m_{i} \in \mathbb{N}, y_{i} \in \mathbb{Z}^{d}, a_{i} \in \mathbb{N}$. The $r$-point functions appear explicitly in this description, since

$$
\mathbb{E}^{(\mathrm{brw})}\left[\prod_{i=1}^{r-1} \mu_{m_{i}}\left(y_{i}\right)^{a_{i}}\right]=\tau_{\vec{n}}(\vec{x}),
$$

where $\left(x_{j}, n_{j}\right)$ equals $\left(y_{i}, m_{i}\right)$ precisely $a_{i}$ times. Thus, the joint moments of the measures $\left\{\mu_{n}\right\}_{n=0}^{\infty}$ are equal to the $r$-point functions. In the remainder of this section, we will give recursive formulas for $\tau_{\vec{n}}(\vec{x})$, and identify the $r$-point functions of IIBRW in terms of those of BRW. We start with the latter. The IIBRW $r$-point functions are defined, for $m_{i} \geq 0$ and $x_{i} \in \mathbb{Z}^{d}$, and similarly to (2.17), by

$$
\rho_{\vec{m}}(\vec{x})=\sum_{i_{1}, \ldots, i_{r-1}} \mathbb{P}_{\infty}^{\text {(brw) }}\left(i_{j} \in T, \phi\left(i_{j}\right)=x_{j},\left|i_{j}\right|=m_{j} \text { for each } j=1, \ldots, r-1\right) .
$$

In the theorem below we identify $\rho_{\vec{m}}(\vec{x})=\rho_{m_{1}, \ldots, m_{r-1}}\left(x_{1}, \ldots, x_{r-1}\right)$ :

Theorem 2.2. For all $\vec{m}=\left(m_{1}, \ldots, m_{r-1}\right)$ with $m_{i} \geq 0$ and $\vec{x}=\left(x_{1}, \ldots, x_{r-1}\right)$ with $x_{i} \in \mathbb{R}^{d}$,

$$
\rho_{m_{1}, \ldots, m_{r-1}}\left(x_{1}, \ldots, x_{r-1}\right)=\sum_{x_{0} \in \mathbb{Z}^{d}} \tau_{\bar{m}, m_{1}, \ldots, m_{r-1}}\left(x_{0}, x_{1}, \ldots, x_{r-1}\right),
$$

where $\bar{m}$ denotes the largest component of $\vec{m}=\left(m_{1}, \ldots, m_{r-1}\right)$.

Proof. To prove (2.23) for branching random walk, we observe that by (2.17) and (2.12) and the fact that the event that $\left\{i_{j} \in T, \phi\left(i_{j}\right)=x_{j},\left|i_{j}\right|=m_{j}\right.$ for each $\left.j=1, \ldots, m-1\right\}$ only depends on $(T, \phi)_{m}$,

$$
\begin{aligned}
& \rho_{m_{1}, \ldots, m_{r-1}}\left(x_{1}, \ldots, x_{r-1}\right) \\
& =\lim _{n \rightarrow \infty} \sum_{i_{1}, \ldots, i_{r-1}} \mathbb{P}_{n}^{\text {(brw) }}\left(i_{j} \in T, \phi\left(i_{j}\right)=x_{j},\left|i_{j}\right|=m_{j} \text { for each } j=1, \ldots, r-1\right) \\
& =\lim _{n \rightarrow \infty} \sum_{i_{1}, \ldots, i_{r-1}} \mathbb{P}_{\bar{m}}^{\text {(brw) }}\left(i_{j} \in T, \phi\left(i_{j}\right)=x_{j},\left|i_{j}\right|=m_{j} \text { for each } j=0, \ldots, r-1\right) \\
& =\sum_{x_{0}} \tau_{\bar{m}, \vec{m}}\left(x_{0}, x_{1}, \ldots, x_{r-1}\right),
\end{aligned}
$$

where in the second equality, we write $m_{0}=\bar{m}$.

By Theorem 2.2, to identify the $r$-point functions of IIBRW, it suffices to identify the $r$-point functions of BRW. We will now investigate the $r$-point functions for BRW. We first introduce some notation. Let $\left(f_{j}\right)_{j=0}^{\infty}$ denote the factorial moments of the distribution $\left(p_{m}\right)_{m=0}^{\infty}$, i.e.,

$$
f_{j}=\sum_{m=j}^{\infty} \frac{m !}{(m-j) !} p_{m}
$$


Also, we write $\mathcal{P}_{j}$ for the number of partitions of $\{1, \ldots, r-1\}$ into $j$ non-empty sets, where we order the elements of $\vec{I} \in \mathcal{P}_{j}$ by ordering the smallest components. Thus, $I_{1}$ contains the element 1. Finally, for $I=\left\{i_{1}, \ldots, i_{j}\right\} \subseteq\{1, \ldots, r-1\}$, we write $\vec{n}_{I}=\left(n_{i_{1}}, \ldots, n_{i_{j}}\right)$. We will prove the following proposition:

Proposition 2.3. For every $\vec{x} \in \mathbb{Z}^{d(r-1)}$ and every $\vec{n}=\left(n_{1}, \ldots, n_{r-1}\right)$ with $n_{i} \geq 1$ for all $i=$ $1, \ldots, r-1$,

$$
\tau_{\vec{n}}(\vec{x})=\sum_{j=1}^{r-1} f_{j} \sum_{\vec{I} \in \mathcal{P}_{j}} \prod_{s=1}^{j}\left(D * \tau_{\vec{n}_{I_{s}}-1}\right)\left(\vec{x}_{I_{s}}\right) .
$$

Before proving Proposition 2.3, we will discuss its relevance. The significance of (2.26) lies in the fact that we can use it recursively to identify the $r$-point functions. As a side remark, we immediately see that when $f_{r-1}=\infty$, then also there exist $x_{1}, \ldots, x_{r-1}$ such that $\tau_{\vec{n}}(\vec{x})=\infty$. For $r=2$, we obtain

$$
\tau_{n}(x)=f_{1}\left(D * \tau_{n-1}\right)(x), \quad \text { so that } \quad \tau_{n}(x)=f_{1}^{n} D^{* n}(x)=D^{* n}(x),
$$

where the last equality holds since the branching process is critical.

A special example arises when we consider binary branching, i.e., $p_{m}=\frac{1}{2}\left(\delta_{m, 0}+\delta_{m, 2}\right)$. In this case, $f_{1}=f_{2}=1$, and $f_{m}=0$ for all $m \geq 3 .{ }^{1}$ Thus, we obtain that, writing $I=I_{2}$, so that $1 \notin I$,

$$
\tau_{\vec{n}}(\vec{x})=\left(D * \tau_{\vec{n}-1}\right)(\vec{x})+\sum_{I \subseteq J_{1}: I \neq \varnothing}\left(D * \tau_{\vec{n}_{I}-1}\right)\left(\vec{x}_{I}\right)\left(D * \tau_{\vec{n}_{J \backslash I}-1}\right)\left(\vec{x}_{J \backslash I}\right),
$$

where $J=\{1, \ldots, r-1\}, J_{1}=J \backslash\{1\}$. Iterating the recursion yields

$$
\tau_{\vec{n}}(\vec{x})=\sum_{I \subseteq J_{1}: I \neq \varnothing} \sum_{m=0}^{n-1} \sum_{y} D^{* m}(y)\left(D * \tau_{\vec{n}_{I}-m-1}\right)\left(\vec{x}_{I}-y\right)\left(D * \tau_{\vec{n}_{J \backslash I}-m-1}\right)\left(\vec{x}_{J \backslash I}-y\right),
$$

where $\underline{n}$ denotes the minimal element of $\vec{n}=\left(n_{1}, \ldots, n_{r-1}\right)$. Using (2.27), we can write (2.30) as

$$
\tau_{\vec{n}}(\vec{x})=\sum_{I \subseteq J_{1}: I \neq \varnothing} \sum_{m=0}^{n-1} \sum_{y} \tau_{m}(y)\left(D * \tau_{\vec{n}_{I}-m-1}\right)\left(\vec{x}_{I}-y\right)\left(D * \tau_{\vec{n}_{J \backslash I}-m-1}\right)\left(\vec{x}_{J \backslash I}-y\right) .
$$

Equation (2.30) yields an explicit recursion for the $r$-point function in terms of $r$, since on the right-hand side only $s$-point functions with $s<r$ appear. For different offspring distributions, (2.26) is not so easily solved, and in Section 3.4 below, we will identify the scaling limit of $\tau_{\vec{n}}(\vec{x})$ for general offspring distributions, by proving that the contribution due to $j \geq 3$ in (2.26) is an error term.

Proof of Theorem 2.3. Recall that

$$
\tau_{n_{1}, \ldots, n_{r-1}}\left(x_{1}, \ldots, x_{r-1}\right)=\sum_{i_{1}, \ldots, i_{r-1}} \mathbb{P}^{\text {(brw })}\left(i_{j} \in T, \phi\left(i_{j}\right)=x_{j},\left|i_{j}\right|=n_{j} \text { for each } j=1, \ldots, r-1\right)
$$

We fix $i_{1}, \ldots, i_{r-1}$ in (2.31). We condition on the number of offspring of the root, and denote this number by $l$. These particles are labeled as $01,02, \ldots, 0 l$. We write, for $i=1, \ldots, l$,

$$
A_{i}=\left\{j: 0 i \longrightarrow i_{j}\right\}
$$

\footnotetext{
${ }^{1}$ The fact that $f_{m}=0$ for all $m \geq 3$ is implied by $p_{m}=0$ for $m \geq 3$, and thus, the computation holds somewhat more generally.
} 
to be the indices that $0 i$ is connected to. Thus, $j \in A_{i}$ precisely when $0 i$ is an ancestor of $i_{j}$. We have that $A_{i} \cap A_{j}=\varnothing$ for $i \neq j$ and $\bigcup_{i=1}^{l} A_{i}=\{1, \ldots, r-1\}$. Denote by $y_{i}$ the spatial location of $0 i$. Then we can write, for each $\mathcal{A}_{1}, \ldots, \mathcal{A}_{l}$,

$$
\begin{aligned}
& \sum_{i_{1}, \ldots, i_{r-1}} \mathbb{P}^{\text {(brw) }}\left(i_{j} \in T, \phi\left(i_{j}\right)=x_{j},\left|i_{j}\right|=n_{j} \forall j=1, \ldots, r-1, A_{t}=\mathcal{A}_{t} \forall t=1, \ldots, l \mid \xi_{0}=l, \phi(0 t)=y_{t}\right) \\
& \quad=\prod_{t=1}^{l} \sum_{i_{j}: j \in \mathcal{A}_{t}} \mathbb{P}^{\text {(brw) }}\left(i_{j} \in T, \phi\left(i_{j}\right)=x_{j}-y_{t},\left|i_{j}\right|=n_{j}-1 \text { for each } j \in \mathcal{A}_{t}\right) \\
& \quad=\prod_{t=1}^{l} \tau_{\vec{n}_{\mathcal{A}_{t}}-1}\left(\vec{x}_{\mathcal{A}_{t}}-y_{t}\right) .
\end{aligned}
$$

Then, we end up with

$$
\begin{aligned}
\tau_{\vec{n}}(\vec{x}) & =\sum_{l=1}^{\infty} \sum_{\vec{A}} \sum_{y_{1}, \ldots, y_{l}}\left[\prod_{t=1}^{l} \tau_{\vec{n}_{A_{t}}-1}\left(\vec{x}_{A_{t}}-y_{t}\right)\right] \mathbb{P}^{(\mathrm{brw})}\left(\xi_{0}=l, \phi(0 t)=y_{t}\right) \\
& =\sum_{l=1}^{\infty} \sum_{\vec{A}} p_{l} \sum_{y_{1}, \ldots, y_{l}} \prod_{t=1}^{l} D\left(y_{t}\right) \tau_{\vec{n}_{A_{t}}-1}\left(\vec{x}_{A_{t}}-y_{t}\right)=\sum_{l=1}^{\infty} \sum_{\vec{A}} p_{l} \prod_{t=1}^{l}\left(D * \tau_{\vec{n}_{A_{t}}-1}\right)\left(\vec{x}_{A_{t}}\right) .
\end{aligned}
$$

We further note that when $A_{i}=\varnothing$, then $\tau_{\vec{n}_{A_{t}}}\left(\vec{n}_{A_{t}}-y_{t}\right)=1$, so that we can restrict the product over $A_{t}$ such that $A_{t} \neq \varnothing$. Let $j$ denote the number of non-empty elements of $A_{t}$, and write $I_{1}, \ldots, I_{j}$ for the non-empty elements of $A_{t}$, ordered in the unique way that $\vec{I} \in \mathcal{P}_{j}$. We can identify $I_{s}=A_{t_{s}}$, so that we have

$$
\prod_{t=1}^{l}\left(D * \tau_{\vec{n}_{A_{t}}-1}\right)\left(\vec{x}_{A_{t}}\right)=\prod_{s=1}^{j}\left(D * \tau_{\vec{n}_{A_{t}}}-1\right)\left(\vec{x}_{A_{t_{s}}}\right)=\prod_{s=1}^{j}\left(D * \tau_{\vec{n}_{I_{s}}-1}\right)\left(\vec{x}_{I_{s}}\right)
$$

Then, the number of different ways of choosing $A_{1}, \ldots, A_{l}$ such that $I_{1}, \ldots, I_{j}$ are fixed equals $\frac{l !}{(l-j) !}$ for each $\vec{I}=\left(I_{1}, \ldots, I_{j}\right) \in \mathcal{P}_{j}$. Thus, we arrive at

$$
\tau_{\vec{n}}(\vec{x})=\sum_{j=1}^{r-1} \sum_{\vec{I} \in \mathcal{P}_{j}} \sum_{l=1}^{\infty} p_{l} \frac{l !}{(l-j) !} \prod_{s=1}^{j}\left(D * \tau_{\vec{n}_{I_{s}}-1}\right)\left(\vec{x}_{I_{s}}\right)=\sum_{j=1}^{r-1} \sum_{\vec{I} \in \mathcal{P}_{j}} f_{j} \prod_{s=1}^{j}\left(D * \tau_{\vec{n}_{I_{s}}-1}\right)\left(\vec{x}_{I_{s}}\right) .
$$

This completes the proof of Proposition 2.3

\subsection{The immortal particle}

In the following theorem, we investigate the number of particles at any given time that have infinitely many offspring. In its statement, and for $i \in T$, we write $i \longrightarrow \infty$ for the statement that the tree $T(i)$ rooted at $i$ is infinite.

Theorem 2.4. Under $\mathbb{P}_{\infty}^{(\text {brw })}$, for every $m$, there is a unique $i \in T$ with $|i|=m$ such that $i \longrightarrow \infty$.

The above result says that there is a single immortal particle. This immortal particle performs a random walk with transition probabilities $D$, and the mass alive at any time is produced along the path of this single particle and performs unconditioned critical branching random walk. Before proving Theorem 2.4, we first explain this immortal particle picture in detail. 
We construct IIBRW in the following way. Let $V_{0}$ be the root of the tree, and let $V_{n}$ be the label of the (unique) child of $V_{0} V_{1} \cdots V_{n-1}$ that has an infinite tree emerging from it. Let $\zeta_{n}$ be the total offspring of $V_{n-1}$. Then, $\left\{\left(V_{n}, \zeta_{n}\right)\right\}_{n=0}^{\infty}$ is an i.i.d. sequence with law

$$
\mathbb{P}_{\infty}^{\text {(brw) }}\left(V_{n}=j, \zeta_{n}=k\right)=p_{k+1} \quad(1 \leq j \leq k+1) .
$$

The (unique) infinite line of decent is now $V_{0}, V_{0} V_{1}, V_{0} V_{1} V_{2}, \ldots$ Embed this infinite paths into $\mathbb{Z}^{d}$ as a single random walk path. Then, at the node $V_{0} V_{1} \cdots V_{n}$ in the infinite path, start $\zeta_{n}$ independent unconditioned branching random walks, for which the root has word $V_{0} V_{1} \cdots V_{n-1} j$ for any $j \neq V_{n}$. The law of the obtained process is equal to the law of $(T, \phi)$ under $\mathbb{P}_{\infty}^{\text {(brw) }}$.

The above construction is quite involved, as we need to keep track of what the infinite line of decent is. If we were only to be interested in the spatial locations of the particles $(\phi(i))_{i \in T}$ rather than in the tree together with the spatial locations of the particles $(T, \phi)$, then the construction simplifies considerably. Indeed, in this case, we create single infinite random walk path $\{\omega(n)\}_{n=0}^{\infty}$ in $\mathbb{Z}^{d}$ and at each position $\omega(n)$, we start $\zeta_{n}$ independent unconditioned branching random walks, where $\left\{\zeta_{n}\right\}_{n=0}^{\infty}$ is an i.i.d. sequence with law

$$
\mathbb{P}_{\infty}^{\text {(brw) }}\left(\zeta_{n}=k\right)=(k+1) p_{k+1} \quad(k \geq 0) .
$$

Therefore, $\zeta_{n}$ is a size-biased version of the law $\left(p_{m}\right)_{m=0}^{\infty}$ minus one, and is the marginal of $\zeta_{n}$ in the law in (2.37). We will discuss the history of this problem in Section 2.4 below.

Proof of Theorem 2.4. Fix $k \geq m$. We write $i \longrightarrow n$ for the event that there exists $j \in T(i)$ with $|j|=n$. Then,

$$
\begin{aligned}
\mathbb{P}_{\infty}^{\text {(brw) }} & \left(\exists i_{1}, i_{2} \in T \text { with }\left|i_{1}\right|=\left|i_{2}\right|=m, i_{1} \neq i_{2}, i_{1}, i_{2} \longrightarrow \infty\right) \\
& \leq \mathbb{P}_{\infty}^{\text {(brw }}\left(\exists i_{1}, i_{2} \in T \text { with }\left|i_{1}\right|=\left|i_{2}\right|=m, i_{1} \neq i_{2}, i_{1}, i_{2} \longrightarrow k\right) \\
& =\lim _{n \rightarrow \infty} \mathbb{Q}_{n}^{\text {(brw }}\left(\exists i_{1}, i_{2} \in T \text { with }\left|i_{1}\right|=\left|i_{2}\right|=m, i_{1} \neq i_{2}, i_{1}, i_{2} \longrightarrow k\right),
\end{aligned}
$$

where we use Theorem 2.1 for the last equality. We now continue to compute

$$
\begin{aligned}
\mathbb{P}_{\infty}^{\text {(brw })} & \left(\exists i_{1}, i_{2} \in T \text { with }\left|i_{1}\right|=\left|i_{2}\right|=m, i_{1} \neq i_{2}, i_{1}, i_{2} \longrightarrow \infty\right) \\
& \leq \lim _{n \rightarrow \infty} \frac{1}{\theta_{n}} \mathbb{P}\left(\exists i_{1}, i_{2} \in T \text { with }\left|i_{1}\right|=\left|i_{2}\right|=m, i_{1} \neq i_{2}, i_{1}, i_{2} \longrightarrow k, \exists i_{0} \text { with }\left|i_{0}\right|=m, i_{0} \longrightarrow n\right) \\
& \leq \lim _{n \rightarrow \infty} \frac{1}{\theta_{n}} \mathbb{P}\left(\exists i_{1}, i_{2} \in T \text { with }\left|i_{1}\right|=\left|i_{2}\right|=m, i_{1} \neq i_{2}, i_{1} \longrightarrow k, i_{2} \longrightarrow n\right) \\
& \leq \lim _{n \rightarrow \infty} \frac{\theta_{n-m} \theta_{k-m}}{\theta_{n}}=\theta_{k-m}
\end{aligned}
$$

where we use the fact that the event that there exist $i_{0}, i_{1}, i_{2}$ with $i_{1} \neq i_{2}$ and such that $i_{0} \longrightarrow$ $n, i_{1}, i_{2} \longrightarrow k$ is contained in the event that there exists distinct $i_{1}, i_{2}$ such that $i_{1} \longrightarrow n$ and $i_{2} \longrightarrow k$. Let $k \rightarrow \infty$ to obtain the result.

\subsection{Discussion and notes}

Our results for incipient infinite branching random walk are not new. For example, the IIBRW measure in (2.11) was already constructed by Kesten 44, who also identified the IIBRW measure in (2.13). See the notes below for more details of Kesten's work. We now give an account of the history of the problem. 
IIBRW corresponds to simply embedding critical branching process trees conditioned on nonextinction in $\mathbb{Z}^{d}$. Critical branching process trees conditioned on non-extinction have a long history, and many properties are known for such processes. For example, the immortal particle picture is present in [24, Page 304], which constructs conditioned branching processes simplifying constructions in 44, 48, 53. The description in (2.37) follows from 24, Lemma 2.1]. The asymptotics in (2.16) is shown in [24, Theorem 3.1], and goes in its most basic form back to Kolmogorov [47. See also the references in 24] for a more detailed account of the history of (2.16).

In [27, critical branching process trees conditioned on non-extinction are constructed for binomial offspring distributions. This corresponds to the incipient infinite clusters for percolation on a tree. We will discuss incipient infinite percolation clusters in more detail in Section 4 and 5.1 .

The immortal particle picture and the fact that there is a unique infinite path for critical branching process trees conditioned on non-extinction, are essential ingredients in [44, where Kesten investigates random walk on a critical branching process conditioned to survive forever. Since the probability that the size of the total progeny for critical branching processes exceeds $n$ decays as $1 / \sqrt{n}$, these critical branching processes create large dead ends. The random walker spends considerable time to get out of these dead ends, which slows the random walk down considerably, and, as a result, the graph distance of the walker after $n$-steps grows subdiffusively as $n^{1 / 3}$. If we were to embed the tree into $\mathbb{Z}^{d}$, this suggests that random walk on the IIBRW has displacement of the order $n^{1 / 6}$.

The use of moment measures to describe BRW is not so common in the probability literature. One reason may be that it they are harder to use for branching laws that have all moments. For instance, the approach in Theorem 2.3 is restricted to measures having all moments. The convergence to SBM, as described in the next section, also holds when, say, the third moment is finite. It may be possible to use moment measures in combination with Laplace transforms to overcome these problems. In the probability community, other methods, such as martingale methods (see e.g., [57]) are used to prove convergence towards SBM. Such methods, however, are based on the fact that BRW has independent branching and motion. The models we will discuss in the sequel are self-interacting, and moment measures are a more robust way to investigate these models.

We have proved that two constructions for IIBRW agree, namely, (1) by size-biasing with respect to $N_{n}$ and letting $n \rightarrow \infty$, and (2) by conditioning on $N_{n}>0$ and letting $n \rightarrow \infty$. We believe that there are many more constructions leading to the same limit. Examples are (3) Conditioning super-critical branching random walk on non-extinction, and taking the limit when the parameter turns to the critical value; (4) Conditioning the tree $T$ to have total size $n$ and taking the limit $n \rightarrow \infty$. It would be of interest to investigate these constructions, and possibly other related constructions, in more detail.

\section{Infinite canonical super-Brownian motion}

In this section, we construct the incipient infinite canonical measure for super-Brownian motion, which we will abbreviate as infinite canonical super-Brownian motion (ICSBM). We will present two constructions, mirroring the two constructions of IIBRW. We will also motivate the constructions and definitions using the discussion of branching random walk in the previous section to make the details comprehensible for non-specialists in the field of super-processes. 


\subsection{Super-Brownian motion and the canonical measure}

We first introduce some notation. We denote by $\mathbb{N}_{0}$ the canonical measure of super-Brownian motion. This canonical measure is a measure on continuous paths from $[0, \infty)$ into non-negative finite measures on $\mathbb{R}^{d}$. The canonical measure is an elusive object, as is it not a probability measure, but rather a $\sigma$-finite, non-negative measure. We take $\mathbb{N}_{0}$ to be normalised to have unit branching and diffusion rates. We will now first discuss a construction of the canonical measure as a scaling limit of branching random walk to explain the canonical measure in more detail. For simplicity, we take an offspring distribution for which $\sigma_{p}=1$. Let $n \geq 0$, and recall the definition of the random measures $\left\{\mu_{n}\right\}_{n=0}^{\infty}$ in (2.19) $-(2.20)$. We expect that, as $n \rightarrow \infty$, the process $\left\{\mu_{n}\right\}_{n=0}^{\infty}$ has a scaling limit. The difficulty in describing this scaling limit, however, is that

$$
\mathbb{P}^{\text {(brw) }}\left(\exists x \in \mathbb{Z}^{d} \text { such that } \mu_{n}(x) \neq 0\right)=\mathbb{P}^{\text {(brw) }}(\exists i \in T:|i|=n)=\theta_{n},
$$

so that by (2.16), with probability close to 1 for $n$ large, the random measure $\mu_{n}$ has mass zero. We are interested in the scaling limit, and, in particular, in large realizations of $T$ for which $\mu_{n}$ is not identically equal to 0 . We now describe the construction of the scaling limit in detail.

We define, for $t \geq 0$ and $x \in \mathbb{R}^{d}$, the random measure-valued Markov process

$$
X_{n, t}(x)=\frac{1}{n} \mu_{\lfloor n t\rfloor}\left(\left\lfloor x \sqrt{\sigma^{2} n}\right\rfloor\right),
$$

where $\left\lfloor x \sqrt{\sigma^{2} n}\right\rfloor=\left(\left\lfloor x_{1} \sqrt{\sigma^{2} n}\right\rfloor, \ldots,\left\lfloor x_{d} \sqrt{\sigma^{2} n}\right\rfloor\right)$.

We first motivate the scaling in (3.2). It turns out that when there is a particle alive at time $\lfloor n t\rfloor$, then there are in fact many particles alive at the same time. Indeed, it can be shown that conditionally on $N_{m} \geq 1$, the random variable $\frac{N_{m}}{m}$ weakly converges to an exponential random variable. See [65] or [57, Theorem II.1.1(b)]. We will be particularly interested in branching processes that are alive at time proportional to $n$, so that we should normalise the number of particles with a factor of $\frac{1}{n}$. This explains the factor $\frac{1}{n}$ in (3.2). To explain the scaling in the spatial coordinate, we note that when there is a particle present at some site $z$ at time proportional to $n$, then this particle has arrived to $z$ by a random walk path of length proportional to $n$. Therefore, we can expect that $z$ is of the order $\sqrt{\sigma^{2} n}$. This explains the scaling in (3.2). We now describe the scaling limit.

For an event $E$ that is a measurable subset of the space of measure-valued paths on $\mathbb{R}^{d}$, we take the limit

$$
\lim _{n \rightarrow \infty} n \mathbb{P}^{\text {(brw) }}\left(\left\{X_{n, t}\right\}_{t \geq 0} \in E\right) .
$$

It turns out that the above limit exists as an element of $[0, \infty]$ (see e.g., [57, Theorem II.7.3(a)]), and is by definition equal to the measure of the indicator of the event $E$ under the canonical measure of super-Brownian motion, i.e., to $\mathbb{N}_{0}(I[E])$. The factor $n$ in (3.3) explains that the measure $\mathbb{N}_{0}$ is not a probability measure, but rather a $\sigma$-finite measure. We now discuss this construction of the canonical measure and its relation to super-Brownian motion started from a proper initial measure.

For a measure $\mu$ on $\mathbb{R}^{d}$, we write $\mu(1)=\int_{\mathbb{R}^{d}} 1 d \mu$ for its total mass. We write $\left\{X_{t}\right\}_{t \geq 0}$ for the process of non-negative measures under the canonical measure $\mathbb{N}_{0}$. Note that when $E=\left\{X_{t}(1)>\right.$ $0\}$, then by (2.16),

$$
\mathbb{N}_{0}\left(I\left[X_{t}(1)>0\right]\right)=\lim _{n \rightarrow \infty} n \mathbb{P}^{(\text {brw })}\left(X_{n, t}(1)>0\right)=\lim _{n \rightarrow \infty} n \theta_{\lfloor n t\rfloor}=\frac{2}{\sigma_{p}^{2} t}=\frac{2}{t},
$$


since we have assumed that $\sigma_{p}=1$. Therefore, $\mathbb{N}_{0}$ is a finite measure on events $E$ that imply that $X_{t}(1)>0$ for some $t>0$.

Often, super-Brownian motion is considered as starting from a proper initial measure. This corresponds to a different scaling limit. Indeed, let the measure $\mu_{0, n}$ be such that $\mu_{0, n}(x)$ takes integer value for every $x \in \mathbb{Z}^{d}$, and let

$$
\nu_{n}(x)=\frac{1}{n} \mu_{0, n}\left(\left\lfloor x \sqrt{\sigma^{2} n}\right\rfloor\right) .
$$

We assume that $\nu_{n}$ is a measure that weakly converges to some limiting measure $\nu$. Then, we let $\left(T_{x}^{(j)}, \phi_{x}^{(j)}\right)$ for $j=1, \ldots, \mu_{0, n}(x)$ be $\mu_{0, n}(x)$ independent branching random walks started at $x$, so that $\phi_{x}^{(j)}(0)=x$, where 0 is the root of the tree $T_{x}^{(j)}$. We now start with several independent branching random walks with starting points given by the initial measure $\mu_{0, n}$. The spatial locations of the branching random walk particles at time $m$ with initial measure $\mu_{n}$ are then given by

$$
\mu_{m, n}(x)=\sum_{y \in \mathbb{Z}^{d}} \sum_{j=1}^{\mu_{0, n}(y)} \sum_{i \in T_{y}^{(j)}:|i|=m} I\left[\phi_{y}^{(j)}(i)=x\right] .
$$

In words, the random variable $\mu_{m, n}(x)$ equals the number of particles that are present at time $m$ at the location $x$ when we start with initial measure $\mu_{0, n}$. Then, we define

$$
X_{n, t}(x)=\frac{1}{n} \mu_{\lfloor n t\rfloor, n}\left(\left\lfloor x \sqrt{\sigma^{2} n}\right\rfloor\right)
$$

Thus, in particular, $X_{n, 0}(x)=\nu_{n}(x)$. Denote the law of $\left\{X_{n, t}\right\}_{t=0}^{\infty}$ by $\mathbb{P}_{\nu_{n}}^{\text {(brw) }}$. Then, the limit

$$
\lim _{n \rightarrow \infty} \mathbb{P}_{\nu_{n}}^{(\text {brw })}\left(\left\{X_{n, t}\right\}_{t \geq 0} \in E\right)
$$

exists. This limit is

$$
\mathbb{P}_{\nu}\left(\left\{X_{t}\right\}_{t \geq 0} \in E\right)
$$

where $\mathbb{P}_{\nu}$ is the law of super-Brownian motion with initial measure $\nu$.

We can think of the law $\mathbb{P}_{\nu_{n}}^{\text {(brw) }}$ as being described by the evolution of independent branching random walk copies, where the copies are located at positions described by the initial measure $\nu_{n}$. In a similar way, we can think of $\mathbb{P}_{\nu}$ as being described by (infinitely) many independent copies of canonical measures according to the initial measure $\nu$ (see e.g., [57, Theorem II.7.2]). This intuitive picture can be made precise by noting that $\mathbb{P}_{\nu}$ is infinitely divisible, and using the general notion of infinitely divisible measures in e.g., 43. In fact, in the terminology of infinite divisible measures, the canonical measure of super-Brownian motion is the canonical measure for the infinitely divisible measure $\mathbb{P}_{\nu}$. See e.g. [14, Section 1.3, and, in particular, Corollary 1.3] for more details.

On the other hand, we can also describe the canonical measure in terms of SBM by using the Markov property. Indeed, the law of $\left\{X_{s+t}\right\}_{t=0}^{\infty}$ given $X_{s}$ is the same as the law of $\left\{X_{t}\right\}_{t=0}^{\infty}$ under $\mathbb{P}_{X_{s}} \cdot{ }^{2}$ This shows that the laws of SBM with a proper initial measure and the canonical measure are intimately connected.

\footnotetext{
${ }^{2}$ This follows immediately from the Markov property valid for BRW, together with the weak convergence in (3.3) and (3.8). See also 14. Theorem 1.4 (iii)], where the Markov property is stated for the canonical measure.
} 


\subsection{The infinite canonical measure of super-Brownian motion}

We are now ready for the definition of ICSBM, which we will think of as the canonical measure conditioned on non-extinction. Fix $t>0$. Let $\mathcal{F}_{s}$ be the minimal $\sigma$-algebra of events such that $\left\{X_{u}\right\}_{0 \leq u \leq s}$ is measurable with respect to $\mathcal{F}_{s}$, i.e., $\mathcal{F}_{s}=\sigma\left(\left\{X_{u}\right\}_{0 \leq u \leq s}\right)$, and we write

$$
\mathcal{F}=\bigcup_{s \geq 0} \mathcal{F}_{s}
$$

Let $E \in \mathcal{F}_{s}$ for some $s<\infty$. Thus, $E$ only depends on $X_{u}$ for all $u \leq s$. We then define the probability measure

$$
\mathbb{P}_{t}(E)=\frac{\mathbb{N}_{0}\left(I[E] X_{t}(1)\right)}{\mathbb{N}_{0}\left(X_{t}(1)\right)},
$$

where the random variable $X_{t}(1)$ is the total mass of super-Brownian motion at time $t$, and where, for an event $E, I[E]$ is the indicator of the event $E$. The measure $\mathbb{P}_{t}$ is size-biased with respect to the total mass at time $t$. The definition in (3.11) is reminiscent of the definition in (2.6).

We also define a second probability measure

$$
\mathbb{Q}_{t}(E)=\frac{\mathbb{N}_{0}\left(I[E] I\left[X_{t}(1)>0\right]\right)}{\mathbb{N}_{0}\left(I\left[X_{t}(1)>0\right]\right)},
$$

which we can think of as the canonical measure of super-Brownian motion conditioned to survive up to time $t$, and which is reminiscent of the definition in (2.10).

The measures $\mathbb{P}_{t}$ and $\mathbb{Q}_{t}$, for large $t$, ensure that the SBM does not have too small mass. Indeed, when $t$ is large, then with probability close to 1 , we have that $X_{t}(1)=0$. On the contrary, for large $t$, SBM must live until time $t$ in both constructions. We now prove that the above two measures converge, when $t \rightarrow \infty$, to a limiting measure, which we will call infinite canonical super-Brownian motion:

Theorem 3.1. When $t \rightarrow \infty$, and for every $E \in \mathcal{F}_{s}$ for any $s \geq 0, \mathbb{P}_{t}(E)$ and $\mathbb{Q}_{t}(E)$ converge to $\mathbb{P}_{\infty}(E)$ and $\mathbb{Q}_{\infty}(E)$. Moreover, $\mathbb{P}_{\infty}$ and $\mathbb{Q}_{\infty}$ extend to probability measures on the $\sigma$-algebra $\mathcal{F}$, and $\mathbb{P}_{\infty}=\mathbb{Q}_{\infty}$.

Proof. The statement in Theorem 3.1 is simplest to prove for $\mathbb{P}_{\infty}$. Indeed, since the total mass $X_{t}(1)$ is a martingale with $X_{0}(1)=1$, we have that (see also [57, Theorem II.7.2 (iii)])

$$
\mathbb{N}_{0}\left(X_{t}(1)\right)=1
$$

and, when $E \in \mathcal{F}_{s}$,

$$
\mathbb{N}_{0}\left(I[E] X_{t}(1)\right)=\mathbb{N}_{0}\left(I[E] X_{s}(1)\right)
$$

Thus, when $E \in \mathcal{F}_{s}$,

$$
\mathbb{P}_{t}(E)=\mathbb{P}_{s}(E),
$$

and the stated convergence is trivial. Since the limiting measure $\mathbb{P}_{\infty}$ is consistent, we can extend it to the full $\sigma$-algebra by Kolmogorov's Extension Theorem (see e.g. 62]). For the second construction, we have to do a bit more work. We note from [57, Theorem II.7.2 (iii)] that

$$
\mathbb{N}_{0}\left(I\left[X_{t}(1)>0\right]\right)=\frac{2}{t},
$$


(see also (3.4) above) so that it suffices to compute $\mathbb{N}_{0}\left(I[E] I\left[X_{t}(1)>0\right]\right)$. We will first prove that

$$
\lim _{t \rightarrow \infty} t \mathbb{P}_{\mu}\left(X_{t}(1)>0\right)=2 \mu(1)
$$

where $\mathbb{P}_{\mu}$ is the law of super-Brownian motion starting from the measure $\mu$. Equation (3.17) follows from the fact that

$$
\mathbb{P}_{\mu}\left(X_{t}(1)>0\right)=1-e^{-2 \mu(1) t^{-1}} .
$$

(See [23, (1.3)], where the factor 2 is absent due to the fact we have assumed the branching rate to be 1, whereas there it is 2. See also [57, (II.5.12)].) Using (3.17), we can write

$$
t \mathbb{N}_{0}\left(I[E] I\left[X_{t}(1)>0\right]\right)=\mathbb{N}_{0}\left(I[E] t \mathbb{P}_{X_{s}}\left(X_{t-s}(1)>0\right)\right) .
$$

Here we use the Markov property for the canonical measure described above. Using (3.18), and the fact that thus, for $t \geq 2 s$,

$$
t \mathbb{E}_{X_{s}}\left[X_{t-s}(1)>0\right] \leq 2 X_{s}(1) \frac{t}{t-s} \leq 4 X_{s}(1)
$$

which is integrable, we obtain by dominated convergence,

$$
\lim _{t \rightarrow \infty} \mathbb{Q}_{t}(E)=\lim _{t \rightarrow \infty} \frac{\mathbb{N}_{0}\left(I[E] I\left[X_{t}(1)>0\right]\right)}{\mathbb{N}_{0}\left(I\left[X_{t}(1)>0\right]\right)}=\lim _{t \rightarrow \infty} \frac{t}{2} \mathbb{N}_{0}\left(I[E] I\left[X_{t}(1)>0\right]\right)=\mathbb{N}_{0}\left(I[E] X_{s}(1)\right)=\mathbb{P}_{\infty}(E)
$$

This completes the proof of Theorem 3.1.

\subsection{Convergence of BRW r-point functions to SBM moment measures}

In this section, we describe the moment measures of the canonical measure of super-Brownian motion. We first discuss what these moment measures are and how they can be characterized. In the next section, we use this characterization to describe the ICSBM moment measures.

As we will explain in more detail below, moment measures describe the finite-dimensional distributions of super-process. Indeed, a measure can be determined by its expectation of a sufficiently rich class of bounded continuous functions. For a random measure $X_{t}$, we can thus determine the law of $X_{t}$ by describing the laws of $X_{t}(f)$ for a sufficiently rich class of continuous functions, where

$$
X_{t}(f)=\int_{\mathbb{R}^{d}} f(x) d X_{t}(x) .
$$

We will be using Fourier transforms, so that we take as a class of continuous functions $\left\{f_{k}\right\}_{k \in \mathbb{R}^{d}}$, where $f_{k}(x)=e^{i k \cdot x}$, and $k \cdot x$ is the inner product between $x$ and $k$. Thus, in order to determine the law of super-Brownian motion, it suffices to know the law of $\left\{X_{s}\left(f_{k}\right)\right\}_{s \geq 0, k \in \mathbb{R}^{d}}$. This law will be uniquely determined by the finite-dimensional distributions $\left\{X_{s_{i}}\left(f_{k_{i}}\right)\right\}_{i=1}^{r}$ for any $s=\left(s_{1}, \ldots, s_{r}\right)$ and $k=\left(k_{1}, \ldots, k_{r}\right)$. These laws, in turn, will be unique determined in terms of the joint moments, for every vector $\left(a_{1}, \ldots, a_{r}\right) \in \mathbb{N}^{r}$,

$$
\mathbb{N}_{0}\left(\prod_{i=1}^{r} X_{s_{i}}\left(f_{k_{i}}\right)^{a_{i}}\right)=\mathbb{N}_{0}\left(\prod_{j=1}^{l} X_{t_{j}}\left(f_{k_{j}}\right)\right)
$$

where $l=a_{1}+\ldots+a_{r}$, and the components of $\left(t_{1}, \ldots, t_{l}\right)$ are equal to $s_{j}$ precisely $a_{j}$ times. Thus, we are lead to investigate

$$
\hat{M}_{\vec{t}}^{(l)}(\vec{k})=\mathbb{N}_{0}\left(\prod_{j=1}^{l} X_{t_{j}}\left(f_{k_{j}}\right)\right)=\mathbb{N}_{0}\left(\int_{\mathbb{R}^{d l}} X_{t_{1}}\left(d x_{1}\right) \cdots X_{t_{l}}\left(d x_{l}\right) \prod_{j=1}^{l} e^{i k_{j} \cdot x_{j}}\right) .
$$


These are the Fourier transforms of the moment measures of the canonical measure of superBrownian motion.

We next give formulas for these moment measures of the canonical measure of super-Brownian motion. We follow the presentation in [32, Section 4]. We will make use of elementary properties of the $\hat{M}_{\vec{t}}^{(l)}(\vec{k})$, which we now summarise. For $l=1$,

$$
\hat{M}_{t}^{(1)}(\vec{k})=e^{-|k|^{2} t / 2 d} .
$$

We will write

$$
k_{I}=\sum_{i \in I} k_{i}, \quad \vec{k}_{I}=\left(k_{i} ; i \in I\right)
$$

For $l>1$, the $\hat{M}_{\vec{t}}^{(l)}(\vec{k})$ are given recursively by

$$
\hat{M}_{\vec{t}}^{(l)}(\vec{k})=\int_{0}^{\underline{t}} d t \hat{M}_{t}^{(1)}\left(k_{J}\right) \sum_{I \subset J_{1}:|I| \geq 1} \hat{M}_{\vec{t}_{I}-t}^{(i)}\left(\vec{k}_{I}\right) \hat{M}_{\vec{t}_{J \backslash I}^{(l-i)}}^{\left(\vec{k}_{J \backslash I}\right),}
$$

where $i=|I|, J=\{1, \ldots, l\}, J_{1}=J \backslash\{1\}, \underline{t}=\min _{i} t_{i}, \vec{t}_{I}$ denotes the vector consisting of the components $t_{i}$ of $\vec{t}$ with $i \in I$, and $\vec{t}_{I}-t$ denotes subtraction of $t$ from each component of $\vec{t}_{I}$ [18]. The explicit solution to the recursive formula (3.27) can be found in [39, (1.25)]. For example,

$$
\hat{M}_{t_{1}, t_{2}}^{(2)}\left(k_{1}, k_{2}\right)=\int_{0}^{t_{1} \wedge t_{2}} d t e^{-\left|k_{1}+k_{2}\right|^{2} t / 2 d} e^{-\left|k_{1}\right|^{2}\left(t_{1}-t\right) / 2 d} e^{-\left|k_{2}\right|^{2}\left(t_{2}-t\right) / 2 d} .
$$

Equation (3.28) is a statement, in Fourier language, that mass arrives at given points $\left(x_{1}, t_{1}\right)$, $\left(x_{2}, t_{2}\right)$ via a Brownian path from the origin that splits into two Brownian paths at a time chosen uniformly from the interval $\left[0, t_{1} \wedge t_{2}\right]$. The recursive formula (3.27) has a related interpretation for all $l \geq 2$, in which $t$ is the time of the first branching. The sets $I$ and $J \backslash I$ label the offspring of each of the two particles after the first branching.

The main result in this section is the proof that the moment measures of the canonical measure of SBM arise as the scaling limits of the BRW $r$-point functions:

Theorem 3.2. Fix an offspring distribution $\left(p_{m}\right)_{m=0}^{\infty}$ such that all moments are finite, and assume that (2.4) holds. Then,

$$
\hat{\tau}_{\vec{n}}\left(\vec{k} / \sqrt{\sigma^{2} n}\right)=\left(\sigma_{p}^{2} n\right)^{r-2}\left[\hat{M}_{\vec{n} / n}^{(r-1)}(\vec{k})+\mathcal{O}\left(\left(n_{(2)}+1\right)^{-\delta}\right)\right] \quad(r \geq 2)
$$

holds uniformly in $n \geq n_{(2)}$, where $n_{(2)}$ denotes the second largest component of $\vec{n}$. In particular, $\sigma_{p}^{2} n \mathbb{P}^{(\mathrm{brw})}\left(X_{n, \frac{\vec{t}}{\sigma_{p}^{2}}} \in \cdot\right)$ converges to $\mathbb{N}_{0}$ in the sense of convergence of finite-dimensional distributions.

Proof. We start from the recursive formula in Proposition 2.3, and rewrite it as

$$
\tau_{\vec{n}}(\vec{x})=\left(D * \tau_{\vec{n}-1}\right)(\vec{x})+\sigma_{p}^{2} \sum_{I \not \supset 1, I \neq \varnothing}\left(D * \tau_{\vec{n}_{I}-1}\right)\left(\vec{x}_{I}\right)\left(D * \tau_{\vec{n}_{J \backslash I}-1}\right)\left(\vec{x}_{J \backslash I}\right)+e_{\vec{n}}(\vec{x}),
$$

where

$$
e_{\vec{n}}(\vec{x})=\sum_{j=3}^{r-1} f_{j} \sum_{\vec{I} \in \mathcal{P}_{j}} \prod_{s=1}^{r-1}\left(D * \tau_{\vec{n}_{I_{s}}-1}\right)\left(\vec{x}_{I_{s}}\right) .
$$

Thus, $e_{\vec{n}}(\vec{x})$ is the contribution to $\tau_{\vec{n}}(\vec{x})$ where there are at least three children of the root that are connected to elements $i_{j}$ with $\left|i_{j}\right|=n_{j}$ and $\phi\left(i_{j}\right)=x_{j}$. Equation (3.30) is a generalization of (2.30), which was only valid for binary branching. In deriving (3.30), we have use that $f_{1}=1, f_{2}=\sigma_{p}^{2}$. 
We will prove that when $\left(p_{m}\right)_{m=0}^{\infty}$ has all moments, then there exists $C_{r}<\infty$ such that

$$
\left|\hat{e}_{\vec{n}}(\vec{k})\right| \leq \sum_{\vec{x}} e_{\vec{n}}(\vec{x}) \leq C_{r}\left(n_{(2)}+1\right)^{r-3} .
$$

We first show that this suffices to prove the statement in Proposition 3.2. Indeed, we iterate (3.30) until the first term disappears. Then we arrive at

$$
\tau_{\vec{n}}(\vec{x})=\sigma_{p}^{2} \sum_{m=0}^{\underline{n}} \sum_{I \not 1, I \neq \varnothing} \sum_{y} D^{* m}(y)\left(D * \tau_{\vec{n}_{I}-m-1}\right)\left(\vec{x}_{I}-y\right)\left(D * \tau_{\vec{n}_{J \backslash I}-m-1}\right)\left(\vec{x}_{J \backslash I}-y\right)+\varphi_{\vec{n}}(\vec{x}),
$$

where

$$
\varphi_{\vec{n}}(\vec{x})=\sum_{m=0}^{\underline{n}} \sum_{y} D^{* m}(y) e_{\vec{n}-m}(\vec{x}-y)
$$

Clearly, by (3.32),

$$
\left|\hat{\varphi}_{\vec{n}}(\vec{k})\right| \leq \sum_{\vec{x}} \varphi_{\vec{n}}(\vec{x}) \leq C_{r} n_{(2)}^{r-2},
$$

so that $\left|\hat{\varphi}_{\vec{n}}(\vec{k})\right|$ is an error term. We take the Fourier transform of (3.33) to obtain

$$
\hat{\tau}_{\vec{n}}(\vec{k})=\sigma_{p}^{2} \sum_{m=0}^{\underline{n}} \sum_{I \not 1, I \neq \varnothing} \hat{D}^{m}\left(k_{J}\right) \hat{D}\left(k_{I}\right) \hat{\tau}_{\vec{n}_{I}-1}\left(\vec{k}_{I}\right) \hat{D}\left(k_{J \backslash I}\right) \hat{\tau}_{\vec{n}_{J \backslash I}-1}\left(\vec{k}_{J \backslash I}\right)+\hat{\varphi}_{\vec{n}}(\vec{k}) .
$$

Equation (3.36) is a discrete version of (3.27), and it can be used to prove by induction on $r$ that

$$
\hat{\tau}_{\vec{n}}\left(\vec{k} / \sqrt{\sigma^{2} n}\right)=\sigma_{p}^{2(r-2)} n^{r-2}\left[\hat{M}_{\vec{n} / n}^{(r-1)}(\vec{k})+\mathcal{O}\left(\left(n_{(2)}+1\right)^{-\delta}\right)\right] \quad(r \geq 2)
$$

holds uniformly in $n \geq n_{(2)}$. For $r=2$, we use (2.27), which implies that $\hat{\tau}_{n}\left(k / \sqrt{\sigma^{2} n}\right)=$ $\hat{D}\left(k / \sqrt{\sigma^{2} n}\right)^{n}$. When (2.4) holds, then $\hat{D}(k)=1-\sigma^{2} \frac{|k|^{2}}{2 d}+\mathcal{O}\left(|k|^{2+2 \delta}\right.$. This immediately implies the claim for $r=2$, and initializes the induction. We will omit the details of the advancement of the induction, which can be found in [39, Section 2.3], where the same computation was performed for oriented percolation. This completes the proof of Proposition 3.2 subject to (3.32). We complete the proof by proving (3.32).

We use (3.33) to prove by induction on $r$ that there exists a $C_{r}<\infty$ such that

$$
\hat{\tau}_{\vec{n}}(\overrightarrow{0})=\sum_{\vec{x}} \tau_{\vec{n}}(\vec{x}) \leq C_{r}\left(n_{(2)}+1\right)^{r-2} .
$$

For $r=2$, the inequality holds, since the left-hand side equals 1 . This initialises the induction.

Without loss of generality, we may assume that $C_{r}$ is non-decreasing in $r$, and that $C_{r} \geq 1$. To advance the induction, we note that when the above claim holds for all $s \leq r-1$, then, using (3.33), we obtain that

$$
\begin{aligned}
\hat{\tau}_{\vec{n}}(\overrightarrow{0}) & \leq \sum_{j=2}^{r-1} \sum_{m=0}^{\underline{n}} f_{j} \sum_{\vec{I} \in \mathcal{P}_{j}} \prod_{s=1}^{r-1} \hat{\tau}_{\vec{n}_{I_{s}}-1}\left(\overrightarrow{0}_{I_{s}}\right) \leq C_{r-1}^{r-1} \sum_{j=2}^{r-1} f_{j} \sum_{\vec{I} \in \mathcal{P}_{j}} \sum_{m=0}^{\underline{n}} \prod_{s=1}^{r-1}\left(n_{(2)}-m\right)^{\left|I_{s}\right|-1} \\
& \leq C_{r-1}^{r-1} \sum_{j=2}^{r-1} f_{j} \sum_{\vec{I} \in \mathcal{P}_{j}}\left(n_{(2)}+1\right)^{\sum_{s=1}^{r-1}\left|I_{s}\right|-j+1} \leq C_{r}\left(n_{(2)}+1\right)^{r-2}
\end{aligned}
$$

where we use that $\left|I_{1}\right|+\ldots+\left|I_{j}\right|=r-1$, and where $C_{r}$ must be chosen appropriately large. A similar computation, where the sum over $j$ starts at $j=3$, proves (3.32). 


\subsection{The moment measures of ICSBM}

In this section, we describe the moment measure of ICSBM.

We write $M_{\infty ; \vec{s}}^{(l)}$ for the moment measures of ICSBM, and $\hat{M}_{\infty ; \vec{s}}^{(l)}(\vec{k})$ for the Fourier transform of the ICSBM moment measures, i.e.,

$$
\hat{M}_{\infty ; \vec{s}}^{(l)}(\vec{k})=\mathbb{E}_{\infty}\left(\int_{\mathbb{R}^{d l}} X_{s_{1}}\left(d x_{1}\right) \cdots X_{s_{l}}\left(d x_{l}\right) \prod_{j=1}^{l} e^{i k_{j} \cdot x_{j}}\right)
$$

where $\vec{s}=\left(s_{1}, \ldots, s_{l}\right)$ with each $s_{i} \in(0, \infty)$, and $\vec{k}=\left(k_{1}, \ldots, k_{l}\right)$ with each $k_{i} \in \mathbb{R}^{d}$.

We can then identify the moment measures of ICSBM as follows:

Theorem 3.3. For every $\vec{s}=\left(s_{1}, \ldots, s_{l}\right)$ with each $s_{i} \in(0, \infty)$, and $\vec{k}=\left(k_{1}, \ldots, k_{l}\right)$ with each $k_{i} \in \mathbb{R}^{d}$,

$$
\hat{M}_{\infty ; \vec{s}}^{(l)}(\vec{k})=\hat{M}_{\bar{s}, \vec{s}}^{(l+1)}\left(0, k_{1}, \ldots, k_{l}\right),
$$

where $\bar{s}=\max _{1 \leq i \leq l} s_{i}$.

Proof. A similar statement as in (3.41) was proved in [32, Lemma 4.2] when $s_{i}=s$ for some $s$. This proof uses induction on $l$. We now prove the more general version of this claim using a simpler martingale proof. We note that the integral

$$
\int_{\mathbb{R}^{d l}} X_{s_{1}}\left(d x_{1}\right) \cdots X_{s_{l}}\left(d x_{l}\right) \prod_{j=1}^{l} e^{i k_{j} \cdot x_{j}}
$$

only depends on $X_{s}$ for $s \leq \bar{s}$. Therefore, by Theorem 3.1 ,

$$
\hat{M}_{\infty ; \vec{s}}^{(l)}(\vec{k})=\lim _{t \rightarrow \infty} \mathbb{E}_{t}\left(\int_{\mathbb{R}^{d l}} X_{s_{1}}\left(d x_{1}\right) \cdots X_{s_{l}}\left(d x_{l}\right) \prod_{j=1}^{l} e^{i k_{j} \cdot x_{j}}\right) .
$$

By (3.15), we have that

$$
\begin{aligned}
\mathbb{E}_{t}\left(\int_{\mathbb{R}^{d l}} X_{s_{1}}\left(d x_{1}\right) \cdots X_{s_{l}}\left(d x_{l}\right) \prod_{j=1}^{l} e^{i k_{j} \cdot x_{j}}\right) & =\mathbb{E}_{\bar{s}}\left(\int_{\mathbb{R}^{d l}} X_{s_{1}}\left(d x_{1}\right) \cdots X_{s_{l}}\left(d x_{l}\right) \prod_{j=1}^{l} e^{i k_{j} \cdot x_{j}}\right) \\
& =\mathbb{E}\left(\int_{\mathbb{R}^{d l}} X_{s_{1}}\left(d x_{1}\right) \cdots X_{s_{l}}\left(d x_{l}\right) X_{\bar{s}}(1) \prod_{j=1}^{l} e^{i k_{j} \cdot x_{j}}\right) \\
& =\hat{M}_{\bar{s}, \vec{s}}^{(l+1)}\left(0, k_{1}, \ldots, k_{l}\right) .
\end{aligned}
$$

for every $t \geq \bar{s}$. This completes the identification of the moment measures of ICSBM.

Note that, in particular,

$$
\hat{M}_{\infty ; s}^{(1)}(k)=\hat{M}_{s, s}^{(2)}(0, k)=s e^{-\frac{k^{2} s}{2 d}}
$$

and

$$
\hat{M}_{\infty ; \vec{s}}^{(2)}(\vec{k})=\int_{0}^{s_{1} \wedge s_{2}}\left(s_{1}+s_{2}-s\right) e^{-\left|k_{1}+k_{2}\right|^{2} s / 2 d} e^{-\left|k_{1}\right|^{2}\left(s_{1}-s\right) / 2 d} e^{-\left|k_{2}\right|^{2}\left(s_{2}-s\right) / 2 d} d s .
$$

We next investigate the total mass under the ICSBM measure.

Theorem 3.4. For $s>0, X_{s}(1)$ is a size-biased exponential random variable with parameter $2 / s$. 
Proof. In 32, Lemma 4.2(c)], it was proved that for $l \geq 0$,

$$
\mathbb{E}_{\infty}\left[X_{s}(1)^{l}\right]=\hat{M}_{s, \ldots, s}^{(l+1)}(\overrightarrow{0})=\mathbb{N}_{0}\left[X_{s}(1)^{l+1}\right]=s^{l} 2^{-l}(l+1) !,
$$

which are the moments of a size-biased exponential random variable. The distribution of the sizebiased exponential random variable is determined by its moments, since its moment generating function has a positive radius of convergence (see [7, Theorem 30.2]). It therefore follows from the moments in (3.46) that $X_{s}(1)$ is a size-biased exponential random variable with parameter 2.

We note that the exact equality in law is due to the fact that we start with the canonical measure of super-Brownian motion. Indeed, in [23, Theorem (iii)], it follows that for a general measure-valued process with a starting measure $\mu$, and conditionally on survival at time $s$, the random variable $X_{s}(1) / s$ converges weakly to a size-biased random variable, rather than being precisely equal to it for all $s$.

\subsection{Convergence of the IIBRW moment measures}

We now turn to the scaling limit of incipient infinite branching random walk. Given the close connections between SBM and critical BRW, it can be expected that the $r$-point functions for IIBRW converge, appropriately scaled, to their continuous analogues for SBM. This is not trivial, since it involves the interchange of the limits defining IIBRW and ICSBM and the scaling limit for IIBRW. The main result is the following theorem:

Theorem 3.5. Fix an offspring distribution $\left(p_{m}\right)_{m=0}^{\infty}$ such that all moments are finite. Then, for all $r \geq 2, \vec{t}=\left(t_{1}, \ldots, t_{r-1}\right) \in \mathbb{R}_{+}^{r-1}$ and $\vec{k} \in \mathbb{R}^{d(r-1)}$, and with $\delta \in(0,1)$ as in (2.4),

$$
\frac{1}{\left(A^{2} V\right)^{r-1}} \hat{\rho}_{m \vec{t}}\left(\vec{k} / \sqrt{\sigma^{2} m}\right)=m^{r-1} \hat{M}_{\infty, \vec{t}}^{(r)}(\vec{k})\left[1+\mathcal{O}\left(m^{-\delta}\right)\right] .
$$

Consequently, when time is rescaled by $m$ and space by $\sqrt{\sigma^{2} m}$, the finite-dimensional distributions of $\mathbb{P}_{\infty}^{\text {(brw) }}$ converge to those of $\mathbb{P}_{\infty}$.

Proof. We prove a more general version of this result, which we can apply later on to other incipient infinite structures. We will prove the following proposition. We will assume that $\tau$ and $\rho$ are functions that are related via (recall (2.23) $)$

$$
\rho_{n_{1}, \ldots, n_{r-1}}\left(x_{1}, \ldots, x_{r-1}\right)=\lim _{n \rightarrow \infty} \sum_{x_{0} \in \mathbb{Z}^{d}} \tau_{n, n_{1}, \ldots, n_{r-1}}\left(x_{0}, x_{1}, \ldots, x_{r-1}\right) .
$$

Proposition 3.6. If there exist constants $A, V, v, \delta$ such that

$$
\hat{\tau}_{\vec{n}}\left(\vec{k} / \sqrt{v \sigma^{2} n}\right)=A\left(A^{2} V\right)^{r-2} n^{r-2}\left[\hat{M}_{\vec{n} / n}^{(r-1)}(\vec{k})+\mathcal{O}\left(\left(n_{(2)}+1\right)^{-\delta}\right)\right] \quad(r \geq 2)
$$

holds uniformly in $n \geq n_{(2)}$, then

$$
\frac{1}{\left(A^{2} V\right)^{r-1}} \hat{\rho}_{m \vec{t}}\left(\vec{k} / \sqrt{v \sigma^{2} m}\right)=m^{r-1} \hat{M}_{\infty, \vec{t}}^{(r)}(0, \vec{k})\left[1+\mathcal{O}\left(m^{-\delta}\right)\right] .
$$

Proof of Proposition 3.6. Equation (3.47) is an immediate consequence of the assumption (3.49), together with the relation in (3.48).

The proof of Theorem 3.5 is now a combination of Proposition 3.6 and Theorem 3.2.

We close this section with a description of the number of particles alive at time $m$ as a corollary to Theorem 3.5. For this, we recall that the size-biased exponential random variable with parameter $\lambda$ has density

$$
f(x)=\lambda^{2} x e^{-\lambda x} \quad(x \geq 0) .
$$


Theorem 3.7. Under $\mathbb{P}_{\infty}^{(\text {brw })}, \frac{N_{m}}{m}$ converges weakly to a size-biased exponential random variable with parameter $\lambda=\frac{2}{\sigma_{p}^{2}}$.

Proof. We compute the moments of $\frac{N_{m}}{m}$ under the measure $\mathbb{P}_{\infty}^{(\text {brw })}$, which equal

$$
m^{-l} \mathbb{E}_{\infty}^{(\text {brw) }}\left[N_{m}^{l}\right]=m^{-l} \hat{\rho}_{m, \ldots, m}(\overrightarrow{0}) .
$$

By Theorem 3.5 the right-hand side converges to $\sigma_{p}^{2 l} \hat{M}_{\infty, \overrightarrow{1}}^{(l)}(\overrightarrow{0})$, which is the $l^{\text {th }}$-moment of a sizebiased exponential random variable (see the proof of Theorem 3.4). Then we can follow the remainder of the proof of Theorem 3.4.

\subsection{The four-dimensional nature of ICSBM}

We next describe the four-dimensional nature of ICSBM. Let

$$
M(R)=\int_{0}^{\infty} X_{s}\left(B_{R}\right) d s,
$$

where $B_{R}$ is the unit ball of radius $R$. Then we have the following scaling result:

Theorem 3.8. Under $\mathbb{P}_{\infty}, M(R)$ has the same law as $R^{4} M(1)$. Moreover, when $d>4, M(1)<\infty$ $\mathbb{P}_{\infty}$-a.s.

Proof. We use the Brownian scaling, which implies that $\left\{R^{-2} X_{R^{2} t}(\cdot R)\right\}_{0 \leq s<\infty}$ has the same law as $\left\{X_{t}(\cdot)\right\}_{0 \leq s<\infty}$. This equality in law can for instance be seen by comparing the moment measures of $\left\{R^{-2} X_{R^{2} t}(\cdot R)\right\}_{0 \leq s<\infty}$, and by proving that these are equal to the ones of $\left\{X_{t}(\cdot)\right\}_{0 \leq s<\infty}$.

Therefore,

$$
M(R)=\int_{0}^{\infty} X_{s}\left(R B_{1}\right) d s=R^{2} \int_{0}^{\infty} X_{R^{2} s}\left(R B_{1}\right) d s \stackrel{d}{=} R^{2} \int_{0}^{\infty} R^{2} X_{s}\left(B_{1}\right) d s=R^{4} M(1) .
$$

The fact that $M(1)$ is finite a.s. when $d>4$ can be deduced from the fact that $\mathbb{E}_{\infty}[M(1)]<\infty$, which follows from the fact that

$$
\mathbb{E}_{\infty}[M(1)]=\mathbb{E}_{\infty}\left[\int_{0}^{\infty} X_{s}\left(B_{1}\right) d s\right]=\int_{0}^{\infty} \mathbb{E}_{\infty}\left[X_{s}\left(B_{1}\right)\right] d s=\int_{0}^{\infty} \int_{B_{1}} s p_{s}(y) d y d s<\infty,
$$

where we used that

$$
\mathbb{E}_{\infty}\left[X_{s}\left(B_{1}\right)\right]=\int_{B_{1}} s p_{s}(y) d y,
$$

which follows from (3.44) and the Fourier-inversion formula.

Theorem 3.8 is important, as it suggests that critical structures can only converge to ICSBM when they are four-dimensional. It is possible to describe the law of $M(1)$ by computing its moments using Theorem 3.3, but we will not do so here.

\subsection{The immortal particle}

In 22], among other things, there is a nice description of a super-processes conditioned on nonextinction, which goes under the name of Evan's immortal particle. Roughly speaking, when we condition a super-process starting from a proper starting distribution to survive up to some time $t$, then we see only the descendants of a finite number of particles. When we then take the limit of $t \rightarrow \infty$, this number of particles becomes one, and we end up with the descendants of the immortal 
particle. Therefore, we can think of ICSBM as being built up from a single particle undergoing Brownian motion, which gives rise to offspring at a fixed rate. This construction is similar in spirit to the one for IIBRW in Theorem 2.4, even though the relation to BRW is not mentioned in [22.

The construction in [22] is for a super-process starting in a proper initial measure. Since we work with the canonical measure of super-Brownian motion, we look at the super-process started with a single particle, and this identifies the immortal particle as the initial particle. Similarly to the setting in Section 2.3 for IIBRW, we again have a unique infinite line of decent, which 'shakes off' mass at constant rate. This mass which is produced along the infinite line of decent behaves as a usual super-Brownian motion. This description can be seen as an alternative construction of ICSBM.

For ICSBM, the immortal particle gives a powerful pictorial description of the moment measures. Indeed, for $x_{1}$ in the support of ICSBM at time $s_{1}$, we can follow back its path until it hits the path of the immortal particle. Both the path until it hits the immortal particle's path, as well as the path of the immortal particle before they meet are Brownian motion paths, and the motion of these two paths will be independent. According to (3.44), the meeting time is uniform on $\left[0, s_{1}\right]$, which explains the factor $s_{1}$ in (3.44). Thus, the moment measure $M^{(1)}$ can be represented as the union of an infinite path (corresponding to the immortal particle), together with a path of length $s_{1}$ which 'hooks up' to the infinite path at a time which is uniform on $\left[0, s_{1}\right]$. We can iterate this procedure for $x_{2}$ in the support of ICSBM at time $s_{2}$, and this will add a second path from $\left(x_{2}, s_{2}\right)$ which will 'hook up' to one of the two paths present for the moment measure $M^{(1)}$. Iterating this procedure for $M^{(r)}$ with $r$ points in the support of ICSBM will create a tree with a single infinite path, and $r$ paths iteratively connected to the union of the infinite path and the previously added paths. This picture will be useful to describe the relation of other models to ICSBM.

\subsection{Discussion and notes}

The construction of ICSBM presented above is within the folklore of the super-processes community. For instance, in [23], conditioning on non-extinction was considered in the context of general measure-valued diffusions starting from a proper initial measure. However, these results do not immediately apply to the canonical measure. See [23] and the references therein. The notion of the canonical measure of super-Brownian motion was first introduced in [20], and further studied in [51], where its existence and some of its properties are proved. See also [14, Theorem 1.4].

It is well-known that for $d>4$, the range of super-Brownian motion is four-dimensional. This was first proved in [13], see also [57, Theorem III.3.9] and the references therein. One would expect that similar estimates as in [57, Theorem III.3.9] are also true for ICSBM.

As far as we know, this is the first time that it was shown that the moment measures of branching random walk converge to those of super-Brownian motion. Many of the ideas for this convergence are taken from [39], where a similar approach was taken for oriented percolation above 4 spatial dimensions. See Section 4 below. In [8], there is a related proof that the $r$-point functions of BRW conditioned to have total mass equal to $n$ converge to the $r$-point functions of integrated super-Brownian excursion (ISE).

The fact that the total mass at a given time is equal to a size-biased exponential distribution can also be understood by (3.15) and the fact that the total mass at a given time $t$ under the canonical measure conditioned to survive at time $t$ is an exponential distribution (see [57, Theorem II.7.2(iii)]). 


\section{The incipient infinite cluster for spread-out oriented per- colation above $4+1$ dimensions}

Since super-Brownian motion arises as the scaling limit in various critical models, one can expect that also the infinite canonical super-Brownian motion arises as a scaling limit. Here we will give an example where we can prove that ICSBM indeed is the scaling limit, namely, for the incipient infinite cluster for spread-out oriented percolation above $4+1$ dimensions. We first discuss the incipient infinite cluster.

In many models, it is known that at the critical value, clusters are finite. For example, it is believed that the critical percolation probability equals 0 . For oriented percolation on $\mathbb{Z}^{d} \times \mathbb{Z}_{+}$, it was shown in [5, 26] that there is no infinite cluster at the critical point. The notion of the incipient infinite percolation cluster (IIC) is an attempt to describe the infinite structure that is emerging but not quite present at the critical point. Various aspects of the IIC are discussed in [1]. We start by defining spread-out oriented percolation.

The spread-out oriented percolation models are defined as follows. Consider the graph with vertices $\mathbb{Z}^{d} \times \mathbb{Z}_{+}$and directed bonds $((x, n),(y, n+1))$, for $n \geq 0$ and $x, y \in \mathbb{Z}^{d}$. Let $D: \mathbb{Z}^{d} \rightarrow[0,1]$ be a fixed function. Let $p \in\left[0,\|D\|_{\infty}^{-1}\right]$, where $\|\cdot\|_{\infty}$ denotes the supremum norm, so that $p D(x) \leq 1$ for all $x$. We associate to each directed bond $((x, n),(y, n+1))$ an independent random variable taking the value 1 with probability $p D(y-x)$ and 0 with probability $1-p D(y-x)$. We say a bond is occupied when the corresponding random variable is 1 , and vacant when the random variable is 0 . Given a configuration of occupied bonds, we say that $(x, n)$ is connected to $(y, m)$, and write $(x, n) \longrightarrow(y, m)$, if there is an oriented path from $(x, n)$ to $(y, m)$ consisting of occupied bonds, or if $(x, n)=(y, m)$. The joint probability distribution of the bond variables will be denoted $\mathbb{P}^{(\mathrm{op})}$, with corresponding expectation denoted $\mathbb{E}^{(\mathrm{op})}$. Note that $p$ is not a probability, but rather equals the expected number of occupied bonds per vertex. We will always work at the critical percolation threshold, i.e., at $p=p_{c}$, and omit subscripts $p_{c}$ from the notation.

The function $D$ will always be assumed to obey the properties of Assumption D of [38]. Assumption $\mathrm{D}$ involves a positive parameter $L$, which serves to spread out the connections, and which we will take to be large. The parameterisation has been chosen in such a way that $p_{c}$ will be asymptotically equal to 1 as $L \rightarrow \infty$. In particular, Assumption D requires that $\sum_{x \in \mathbb{Z}^{d}} D(x)=1$, that $D(x) \leq C L^{-d}$ for all $x$, and that $C_{1} L \leq \sigma \leq C_{2} L$ (recall (2.3) $)$.

A simple example is

$$
D(x)= \begin{cases}\frac{1}{(2 L+1)^{d}-1} & 0<\|x\|_{\infty} \leq L \\ 0 & \text { otherwise }\end{cases}
$$

for which bonds are of the form $((x, n),(y, n+1))$ with $\|x-y\|_{\infty} \leq L$, and a bond is occupied with probability $p\left[(2 L+1)^{d}-1\right]^{-1}$.

Let $\mathcal{F}$ denote the $\sigma$-algebra of events. A cylinder event is an event that is determined by the occupation status of a finite set of bonds. We denote the algebra of cylinder events by $\mathcal{F}_{0}$. Then $\mathcal{F}$ is the $\sigma$-algebra generated by $\mathcal{F}_{0}$. For our first definition of the IIC, we begin by defining $\mathbb{P}_{n}^{(\mathrm{op})}$ by

$$
\mathbb{P}_{n}^{(\mathrm{op})}(E)=\frac{1}{\tau_{n}^{(\mathrm{op})}} \sum_{x \in \mathbb{Z}^{d}} \mathbb{P}^{(\mathrm{op})}(E \cap\{(0,0) \longrightarrow(x, n)\}) \quad\left(E \in \mathcal{F}_{0}\right),
$$

where $\tau_{n}^{(\mathrm{op})}=\sum_{x \in \mathbb{Z}^{d}} \tau_{n}^{(\mathrm{op})}(x)$ with $\tau_{n}^{(\mathrm{op})}(x)=\mathbb{P}^{(\mathrm{op})}((0,0) \longrightarrow(x, n))$. We then define $\mathbb{P}_{\infty}^{(\mathrm{op})}$ by setting

$$
\mathbb{P}_{\infty}^{(\mathrm{op})}(E)=\lim _{n \rightarrow \infty} \mathbb{P}_{n}^{(\mathrm{op})}(E) \quad\left(E \in \mathcal{F}_{0}\right)
$$


assuming the limit exists. We now turn to the second construction of the incipient infinite cluster. For this, let

$$
S_{n}=\{(0,0) \longrightarrow n\}=\left\{(0,0) \longrightarrow(x, n) \text { for some } x \in \mathbb{Z}^{d}\right\}
$$

denote the event that the cluster of the origin survives to time $n$. Define $\mathbb{Q}_{n}$ by

$$
\mathbb{Q}_{n}^{(\mathrm{op})}(E)=\mathbb{P}^{(\mathrm{op})}\left(E \mid S_{n}\right) \quad\left(E \in \mathcal{F}_{0}\right) .
$$

We then define $\mathbb{Q}_{\infty}^{(\text {op })}$ by setting

$$
\mathbb{Q}_{\infty}^{(\mathrm{op})}(E)=\lim _{n \rightarrow \infty} \mathbb{Q}_{n}^{(\mathrm{op})}(E) \quad\left(E \in \mathcal{F}_{0}\right),
$$

assuming the limit exists.

The following theorem shows that this definition produces a probability measure on $\mathcal{F}$ under which the origin is almost surely connected to infinity. The theorem below is the main result in 32. In its statement, we write

$$
\theta_{n}^{(\mathrm{op})}=\mathbb{P}^{(\mathrm{op})}\left(S_{n}\right) .
$$

Theorem 4.1. Let $d+1>4+1$ and $p=p_{c}$. There $i s$ an $L_{0}=L_{0}(d)$ such that for $L \geq L_{0}$, the limit in (4.3) exists for every cylinder event $E \in \mathcal{F}_{0}$. Moreover, $\mathbb{P}_{\infty}^{(\mathrm{op})}$ extends to a probability measure on the $\sigma$-algebra $\mathcal{F}$, and the origin is almost surely connected to infinity under $\mathbb{P}_{\infty}^{(\text {op) }}$.

If we further assume that there is a finite positive constant $B$ such that

$$
\lim _{n \rightarrow \infty} n \theta_{n}^{(\mathrm{op})}=1 / B
$$

then also the limit in (4.6) exists and $\mathbb{Q}_{\infty}^{(\mathrm{op})}=\mathbb{P}_{\infty}^{(\mathrm{op})}$.

In 35], there is a third construction of the IIC, where we take $p<p_{c}$, and define

$$
\mathbb{Q}_{p}^{(\mathrm{op})}(E)=\frac{1}{\chi^{(\mathrm{op})}(p)} \sum_{(x, n) \in \mathbb{Z}^{d} \times \mathbb{Z}_{+}} \mathbb{P}_{p}^{(\mathrm{op})}(E \cap\{(0,0) \longrightarrow(x, n)\}),
$$

where

$$
\chi^{(\mathrm{op})}(p)=\sum_{(x, n) \in \mathbb{Z}^{d} \times \mathbb{Z}_{+}} \mathbb{P}_{p}^{(\mathrm{op})}((0,0) \longrightarrow(x, n))
$$

denotes the oriented percolation susceptibility, and we now explicitly use the subscript $p<p_{c}$ to indicate the percolation parameter. In [35], it is proved that when $p \uparrow p_{c}$, then $\mathbb{Q}_{p}^{(\mathrm{op})}$ converges to $\mathbb{P}_{\infty}^{(\mathrm{op})}$. This definition works both for oriented and unoriented percolation (see Section [5.1).

\subsection{Convergence of oriented percolation moment measures}

In [39], it was shown that the finite dimensional distributions of the rescaled oriented percolation cluster converge to the ones of super-Brownian motion. We will review this result here. We first define an analogue random measure valued-process $X_{n, t}$ on $\mathbb{R}^{d l}$ by placing mass $\left(A^{2} V n\right)^{-1}$ at each site at times $\lfloor n t\rfloor$ in $\left(v \sigma^{2} n\right)^{-1 / 2} C(0,0)$, i.e., for any subset $E$ of $\mathbb{R}^{d}$,

$$
X_{n, t}(E)=\frac{1}{A^{2} V n} \sum_{x \in\left(v \sigma^{2} n\right)^{1 / 2} E} I[(x,\lfloor n t\rfloor) \in C(0,0)] .
$$

Here $v \sigma^{2}$ serves as the variance of occupied oriented percolation paths. The main result in [39] is the following: 
Theorem 4.2. Let $d+1>4+1$. There exist constants $A, V, v, \delta \in(0,1)$ and an $L_{0}=L_{0}(d)$ such that for $L \geq L_{0}$, and for all $r \geq 2$ and $\vec{k} \in \mathbb{R}^{d(r-1)}$,

$$
\hat{\tau}_{\vec{n}}^{(\mathrm{op})}\left(\vec{k} / \sqrt{v \sigma^{2} n}\right)=A\left(A^{2} V\right)^{r-2} n^{r-2}\left[\hat{M}_{\vec{n} / n}^{(r-1)}(\vec{k})+\mathcal{O}\left(\left(n_{(2)}+1\right)^{-\delta}\right)\right] \quad(r \geq 2)
$$

holds uniformly in $n \geq n_{(2)}$. Consequently, $A V n \mathbb{P}\left(X_{n, \vec{t}} \in \cdot\right)$ converges to $\mathbb{N}_{0}$ in the sense of convergence of finite-dimensional distributions.

For a review of the proof of Theorem 4.2 see [38, Section 2]. The approach can be used more generally. For example, the approach taken in [37] for the contact process above 4 dimensions, or the approach taken in [40] for lattice trees, are based upon the same ideas. We next discuss the convergence of the oriented percolation IIC $r$-point functions.

The oriented percolation IIC $r$-point functions are defined, for $n_{i} \geq 0$ and $x_{i} \in \mathbb{Z}^{d}$, by

$$
\rho_{n_{1}, \ldots, n_{r-1}}^{(\mathrm{op})}\left(x_{1}, \ldots, x_{r-1}\right)=\mathbb{P}_{\infty}^{(\mathrm{op})}\left((0,0) \longrightarrow\left(x_{i}, n_{i}\right) \text { for each } i=1, \ldots, r-1\right) .
$$

We now turn to the scaling limit of the oriented percolation IIC. The main result, which is a direct consequence of results in [32] and 39], is the following theorem:

Theorem 4.3. Let $d+1>4+1$. There is an $L_{0}=L_{0}(d)$ such that for $L \geq L_{0}$, and for all $r \geq 2$, $\vec{t}=\left(t_{1}, \ldots, t_{r-1}\right) \in(0,1]^{r-1}$ and $\vec{k} \in \mathbb{R}^{d(r-1)}$

$$
\frac{1}{\left(A^{2} V\right)^{r-1}} \hat{\rho}_{m \vec{t}}^{(\mathrm{op})}\left(\vec{k} / \sqrt{v \sigma^{2} m}\right)=m^{r-1} \hat{M}_{\infty, \vec{t}}^{(r)}(\vec{k})\left[1+\mathcal{O}\left(m^{-\delta}\right)\right] .
$$

Consequently, when time is rescaled by $m$ and space by $\sqrt{v \sigma^{2} m}$, then the finite-dimensional distributions of $\mathbb{P}_{\infty}^{(\mathrm{op})}$ converge to those of $\mathbb{P}_{\infty}$.

Proof. We will use Proposition [3.6, for which the main assumption is proved in [39, (2.52)]. See Theorem 4.2 above.

In [32], there are more properties of the IIC. For instance, a version of Theorem 3.4 is proved there, as well as a result on the four-dimensional nature that we state now. In order to be able to state the result, we let

$$
C(0,0)=\left\{(y, m) \in \mathbb{Z}^{d} \times \mathbb{Z}_{+}:(0,0) \longrightarrow(y, m)\right\}
$$

denote the connected cluster of the origin, and let

$$
M^{(\mathrm{op})}(R)=\#\{(y, m) \in C(0,0):|y| \leq R\}
$$

denote the total number of sites in the cluster of the origin that are at most a distance $R$ away from the origin, under $\mathbb{P}_{\infty}$. We note that $M^{(\mathrm{op})}(R)$ is the equivalent of $M(R)$ defined in (3.53). In [32, the random variable $M^{(\mathrm{op})}(R)$ has not been studie, but rather its expected value.

Theorem 4.4. Let $d+1>4+1$ and $p=p_{c}$. There are $L_{0}=L_{0}(d)$ and $C_{i}=C_{i}(L, d)>0$ such that for $L \geq L_{0}$,

$$
C_{1} R^{4} \leq \mathbb{E}_{\infty}^{(\mathrm{op})}\left[M^{(\mathrm{op})}(R)\right] \leq C_{2} R^{4}
$$

Theorem 4.4 is a sign that the IIC is four-dimensional.

We complete this section by showing that essentially there is a unique path tending to infinity, meaning that any two infinite paths share bonds under $\mathbb{P}_{\infty}^{(\mathrm{op})}$. This is the equivalent of the immortal particle for ICSBM and IIBRW. Of course, for oriented percolation, there will be many small doubly 
connected parts or sausages along any path to infinity, so that we cannot expect there to be a unique infinite path as for IIBRW in Theorem 2.4.

Before stating the result, we need some definitions. We say that the events $\left\{\left(y_{1}, m_{1}\right) \longrightarrow\right.$ $\left.\left(x_{1}, n_{1}\right)\right\}$ and $\left\{\left(y_{2}, m_{2}\right) \longrightarrow\left(x_{2}, n_{2}\right)\right\}$ occur disjointly, if there exist bond disjoint occupied paths connecting $\left(y_{1}, m_{1}\right)$ to $\left(x_{1}, n_{1}\right)$ and $\left(y_{2}, m_{2}\right)$ to $\left(x_{2}, n_{2}\right)$. We write $\left\{\left(y_{1}, m_{1}\right) \longrightarrow n\right\}$ occurs disjointly from $\left\{\left(y_{2}, m_{2}\right) \longrightarrow n\right\}$ for $n \geq m_{1} \vee m_{2}$ when there exist $x_{1}, x_{2}$ such that the events $\left\{\left(y_{1}, m_{1}\right) \longrightarrow\right.$ $\left.\left(x_{1}, n\right)\right\}$ and $\left\{\left(y_{2}, m_{2}\right) \longrightarrow\left(x_{2}, n\right)\right\}$ occur disjointly. We abbreviate this event by $\left\{\left(y_{1}, m_{1}\right) \longrightarrow\right.$ $n\} \circ\left\{\left(y_{2}, m_{2}\right) \longrightarrow n\right\}$ for $n \in \mathbb{N} \cup\{\infty\}$, where $\left\{\left(y_{1}, m_{1}\right) \longrightarrow \infty\right\} \circ\left\{\left(y_{2}, m_{2}\right) \longrightarrow \infty\right\}$ is the intersection of $\left\{\left(y_{1}, m_{1}\right) \longrightarrow n\right\} \circ\left\{\left(y_{2}, m_{2}\right) \longrightarrow n\right\}$ for all $n$.

Theorem 4.5. Under $\mathbb{P}_{\infty}^{(\mathrm{op})}$, for every $m$, the probability that there exist $y_{1}, y_{2} \in \mathbb{Z}^{d}$ such that $\left(y_{1}, m\right) \longrightarrow \infty$ occurs disjointly from $\left(y_{2}, m\right) \longrightarrow \infty$ is zero.

Proof. We bound, using the BK-inequality,

$$
\begin{aligned}
\mathbb{P}_{\infty}^{(\mathrm{op})}( & \left.\left\{\left(y_{1}, m\right) \longrightarrow \infty\right\} \circ\left\{\left(y_{2}, m\right) \longrightarrow \infty\right\}\right) \\
& =\lim _{k \rightarrow \infty} \mathbb{P}_{\infty}^{(\mathrm{op})}\left(\left\{\left(y_{1}, m\right) \longrightarrow k\right\} \circ\left\{\left(y_{2}, m\right) \longrightarrow k\right\}\right) \\
& =\lim _{k \rightarrow \infty} \lim _{n \rightarrow \infty} \mathbb{Q}_{n}^{(\mathrm{op})}\left(\left\{\left(y_{1}, m\right) \longrightarrow k\right\} \circ\left\{\left(y_{2}, m\right) \longrightarrow k\right\}\right) \\
& =\lim _{k \rightarrow \infty} \lim _{n \rightarrow \infty} \frac{1}{\theta_{n}} \mathbb{P}^{(\mathrm{op})}\left(\left(\left\{\left(y_{1}, m\right) \longrightarrow k\right\} \circ\left\{\left(y_{2}, m\right) \longrightarrow k\right\}\right) \cap\{(0,0) \longrightarrow n\}\right) \\
& \leq \lim _{k \rightarrow \infty} \lim _{n \rightarrow \infty} \frac{2 \theta_{n} \theta_{k-m}}{\theta_{n}}=0,
\end{aligned}
$$

where we use the fact that

$$
\begin{aligned}
& \left(\left\{\left(y_{1}, m\right) \longrightarrow k\right\} \circ\left\{\left(y_{2}, m\right) \longrightarrow k\right\}\right) \cap\{(0,0) \longrightarrow n\} \\
& \quad \subseteq\left(\left\{\left(y_{1}, m\right) \longrightarrow k\right\} \circ\{(0,0) \longrightarrow n\}\right) \cup\left(\left\{\left(y_{2}, m\right) \longrightarrow k\right\} \circ\{(0,0) \longrightarrow n\}\right) .
\end{aligned}
$$

Since the event that there exist $y_{1}, y_{2}$ such that $\left(y_{1}, m\right) \longrightarrow \infty$ occurs disjointly from $\left(y_{2}, m\right) \longrightarrow \infty$ is a countable union of events with probability 0 , the claim follows.

\section{Conjectured scaling to ICSBM: Incipient structures}

In this section, we describe several models of incipient infinite structures where one can expect convergence to ICSBM to hold.

\subsection{The incipient infinite cluster for percolation above 6 dimensions}

For general background on percolation, see [25]. Our models are defined in terms of a function $D: \mathbb{Z}^{d} \rightarrow[0,1]$. Let $p \in\left[0,\|D\|_{\infty}^{-1}\right]$ be a parameter, so that again $p D(x) \leq 1$ for all $x$. We declare a bond $\{u, v\}$ to be occupied with probability $p D(v-u)$ and vacant with probability $1-p D(v-u)$. The occupation status of all bonds are independent random variables. For the nearest-neighbor model, we take $D(x)=1 /(2 d)$ for all $x$ with $|x|=1$, so that each bond is occupied with probability $p /(2 d)$. For the spread-out model, we assume that the conditions in [28, Definition 1.1] are satisfied. The function in (4.1) does obey the assumptions.

The law of the configuration of occupied bonds (at the critical percolation threshold) is denoted by $\mathbb{P}^{(\mathrm{pe})}$ with corresponding expectation denoted by $\mathbb{E}^{(\mathrm{pe})}$. Given a configuration we say that $x$ is connected to $y$, and write $x \longleftrightarrow y$, if there is a path of occupied bonds from $x$ to $y$ (or if $x=y$ ). 
Let $\mathcal{F}$ denote the $\sigma$-algebra of events. A cylinder event is an event given by conditions on the states of finitely many bonds only. We denote the algebra of cylinder events by $\mathcal{F}_{0}$. We define

$$
\mathbb{P}_{x}^{(\mathrm{pe})}(F)=\mathbb{P}^{(\mathrm{pe})}(F \mid 0 \longleftrightarrow x)=\frac{1}{\tau^{(\mathrm{pe})}(x)} \mathbb{P}^{(\mathrm{pe})}(F, 0 \longleftrightarrow x), \quad F \in \mathcal{F}
$$

where $\tau^{(\mathrm{pe})}(x)=\mathbb{P}^{(\mathrm{pe})}(0 \longleftrightarrow x)$. The main result in 35] is the following theorem:

Theorem 5.1. Let $d>6$ and $p=p_{c}$. There is an $L_{0}=L_{0}(d)$ such that for $L \geq L_{0}$ in the spread-out model, the limit

$$
\mathbb{P}_{\infty}^{(\mathrm{pe})}(F)=\lim _{|x| \rightarrow \infty} \mathbb{P}_{x}^{(\mathrm{pe})}(F)
$$

exists for any cylinder event $F$. Also, $\mathbb{P}_{\infty}^{(\mathrm{pe})}$ extends uniquely from $\mathcal{F}_{0}$ to a probability measure on $\mathcal{F}$.

Theorem 5.1 is similar to the existence statement of the IIC for spread-out oriented percolation above $4+1$ dimensions in Theorem 4.1. Moreover, the definition in (4.9) is also proved to exist in [35], and to give the same result. In [35] some properties of $\mathbb{P}_{\infty}^{(\mathrm{pe})}$ were proved, which are the natural equivalents of Theorems 4.4 4.5.

We next turn to the conjecture linking the unoriented percolation IIC to ICSBM. Of course, there is no explicit time variable in unoriented percolation, so will will introduce a natural candidate for a time variable. Define $\operatorname{SP}(x, y)$ to be the shortest path along occupied bonds between $x$ and $y$, and let $|\mathrm{SP}(x, y)|$ be the number of bonds in this shortest path. When $x$ and $y$ are not connected, then we set $|\mathrm{SP}(x, y)|=\infty$. Let $\mathrm{SP}(x)=\mathrm{SP}(0, x)$. We then think of $|\mathrm{SP}(x)|$ as being a time variable analogous to the time variable $n$ in oriented percolation. Define

$$
\tau_{\vec{n}}^{(\mathrm{pe})}(\vec{x})=\mathbb{P}^{(\mathrm{pe})}\left(\left|\mathrm{SP}\left(x_{j}\right)\right|=n_{j} \text { for each } j=1, \ldots, r-1\right)
$$

and

$$
\rho_{\vec{n}}^{(\mathrm{pe})}(\vec{x})=\mathbb{P}_{\infty}^{(\mathrm{pe})}\left(\left|\mathrm{SP}\left(x_{j}\right)\right|=n_{j} \text { for each } j=1, \ldots, r-1\right) .
$$

Then $\tau_{\vec{n}}^{(\mathrm{pe})}(\vec{x})$ is analogous to the oriented percolation probability $\tau_{\vec{n}}^{(\mathrm{op})}(\vec{x})$ of (4.13), while $\rho_{\vec{n}}^{(\mathrm{pe})}(\vec{x})$ is analogous to $\rho_{\vec{n}}^{(\mathrm{op})}(\vec{x})$. Then we conjecture that $\rho_{\vec{n}}^{(\mathrm{pe})}$ converges to the moment measures of ICSBM:

Conjecture 5.2. Let $d>6$. For all $r \geq 2, \vec{t}=\left(t_{1}, \ldots, t_{r-1}\right) \in \mathbb{R}^{r-1}$ and $\vec{k} \in \mathbb{R}^{d(r-1)}$, there exist constants $A, V, v$ and $\delta \in(0,1)$ such that (3.47) holds for $\rho^{(\mathrm{pe})}$.

In order to prove Conjecture [5.2, the key step is to prove a version of (3.49) for unoriented percolation, as conjectured in [39, Section 1.3.3].

\subsection{The contact process incipient infinite cluster above 4 dimensions}

For a general introduction to the contact process, see [52]. We define the spread-out contact process as follows. Let $\mathbf{C}_{t} \subset \mathbb{Z}^{d}$ be the set of infected individuals at time $t \in \mathbb{R}_{+}$, and let $\mathbf{C}_{0}=\{0\}$. An infected site $x$ recovers in a small time interval $[t, t+\varepsilon]$ with probability $\varepsilon+o(\varepsilon)$ independently of $t$, where $o(\varepsilon)$ is a function that satisfies $\lim _{\varepsilon \downarrow 0} O(\varepsilon) / \varepsilon=0$. In other words, $x \in \mathbf{C}_{t}$ recovers with rate 1 . A healthy site $x$ gets infected, depending on the status of its neighboring sites, with rate $\lambda \sum_{y \in \mathbf{C}_{t}} D(x-y)$, where $\lambda \geq 0$ is the infection rate. We denote the associated probability measure by $\mathbb{P}^{\lambda}$. We will assume that the function $D: \mathbb{Z}^{d} \mapsto[0,1]$ is a probability distribution which satisfies the assumptions in Section 4 . 
We will always investigate the contact process at the critical value $\lambda_{c}$ for the sufficiently spreadout contact process above 4 dimensions. In [36, 37, the contact process is investigated and the goal is to prove a version of (3.49) for the contact process. For this, its close analogy to oriented percolation is essentially used. We now explain this connection.

The contact process can be constructed using a graphical representation. We consider $\mathbb{Z}^{d} \times \mathbb{R}_{+}$ as space-time. Along each time line $\{x\} \times \mathbb{R}_{+}$, we place points according to a Poisson process with intensity 1, independently of the other time lines. For each ordered pair of distinct time lines from $\{x\} \times \mathbb{R}_{+}$to $\{y\} \times \mathbb{R}_{+}$, we place directed bonds $((x, t),(y, t)), t \geq 0$, according to a Poisson process with intensity $\lambda D(y-x)$, independently of the other Poisson processes. A site $(x, s)$ is said to be connected to $(y, t)$ if either $(x, s)=(y, t)$ or there is a non-zero path in $\mathbb{Z}^{d} \times \mathbb{R}_{+}$from $(x, s)$ to $(y, t)$ using the Poisson bonds and time line segments traversed in the increasing time direction without traversing the Poisson points. The law of $\mathbf{C}_{t}$ defined above is equal to that of $\left\{x \in \mathbb{Z}^{d}:(0,0)\right.$ is connected to $\left.(x, t)\right\}$.

Inspired by this percolation structure in space-time and following [59], we consider the following oriented percolation process in $\mathbb{Z}^{d} \times \varepsilon \mathbb{Z}_{+}$with $\varepsilon \in(0,1]$ being a discretization parameter. A directed pair $b=((x, t),(y, t+\varepsilon))$ of sites in $\mathbb{Z}^{d} \times \varepsilon \mathbb{Z}_{+}$is called a bond. Each bond is either occupied or vacant independently of the other bonds, and a bond $b=((x, t),(y, t+\varepsilon))$ is occupied with probability

$$
p_{\varepsilon}(y-x)= \begin{cases}1-\varepsilon, & \text { if } x=y, \\ \lambda \varepsilon D(y-x), & \text { otherwise }\end{cases}
$$

provided that $\sup _{x} p_{\varepsilon}(x) \leq 1$. We denote the associated probability measure by $\mathbb{P}_{\varepsilon}^{\lambda}$. It is proved in [6] that $\mathbb{P}_{\varepsilon}^{\lambda}$ weakly converges to $\mathbb{P}^{\lambda}$ as $\varepsilon \downarrow 0$.

Existence of the contact process IIC has not yet been established. The proof in [32] applies to the discretized contact process, and therefore, the only thing left to do is to take the limit $\varepsilon \downarrow 0$. The continuum limit results in [36] can hopefully show that this continuum limit exists. Once the existence of the contact process IIC has been established, convergence of the finite-dimensional distributions towards the moments measures of ICSBM will follow from the results in [36].

\subsection{Incipient infinite lattice trees above 8 dimensions}

There are many connections between lattice trees and super-Brownian motion. In [15, 16, it was shown that the $r$-point functions of lattice trees of fixed size, converge to those of ISE. The statements are complete when dealing with the $r$-point functions where the number of steps between 0 and $x$ along the tree is not fixed, and there are partial results when this number is fixed and scales with the size of the lattice tree.

There is current progress in understanding the connection to SBM [40, when the set-up is somewhat different. Let us introduce some notation. A lattice tree is a tree embedded in $\mathbb{Z}^{d}$ containing no cycles. We give uniform weight to lattice trees with a fixed number of bonds, and assume that the bonds are either nearest-neighbour, or spread-out (as in (4.11)). In general, the number of lattice trees of fixed size grows exponentially with the size. Denote by $\tau^{(1 t)}(N)$ the total number of lattice trees of size $N$ containing 0 . Then, we know that

$$
\lim _{N \rightarrow \infty} \tau^{(\mathrm{lt})}(N)^{1 / N}=\lambda \in(0, \infty)
$$

We define

$$
\tau_{\vec{n}}^{(1 \mathrm{t})}(\vec{x})=\sum_{N=1}^{\infty} \tau_{\vec{n}}^{(1 \mathrm{tt})}(\vec{x} ; N) \lambda^{-N}
$$


to be the $r$-point function for lattice trees, where $\tau_{\vec{n}}^{(\mathrm{lt})}(\vec{x} ; N)$ is the number of lattice trees of size $N$ such that $\left|\mathrm{SP}\left(x_{j}\right)\right|=n_{j}$ for all $j=1, \ldots, r-1$. Of course, existence of the sum in (5.7) is a non-trivial result, and follows from [28] for the spread-out model. The main work in [40] involves the proof that $\tau_{\vec{n}}^{(1 \mathrm{t})}(\vec{x})$ scales to the $r$-point functions of SBM, and therefore satisfies the main assumption in (3.49). If this is completed, it is natural to conjecture that there exists an infinite tree measure, and that this infinite tree measure has ICSBM as a scaling limit. The simplest way to obtain the infinite tree measure is to take the limit

$$
\mathbb{P}_{\infty}^{(1 \mathrm{t})}(E)=\lim _{n \rightarrow \infty} \frac{\sum_{x} \tau_{n}^{(\mathrm{lt})}(x, E)}{\sum_{x} \tau_{n}^{(\mathrm{lt})}(x)},
$$

where, for an event $E$,

$$
\tau_{n}^{(1 \mathrm{tt})}(x, E)=\sum_{N=1}^{\infty} \tau_{n}^{(\mathrm{lt})}(x, E ; N) \lambda^{-N},
$$

and $\tau_{n}^{(1 \mathrm{tt})}(x, E ; N)$ is the number of lattice trees of size $N$ such that $|\mathrm{SP}(x)|=n$ and such that $E$ is satisfied. $\mathbb{P}_{\infty}^{(1 t)}$ is a version of $\mathbb{P}_{t}$ in (3.11). We next state a version of $\mathbb{Q}_{t}$ in (3.12). For this, we let $\tau_{n}^{(1 \mathrm{t})}(E ; N)$ denote the number of lattice trees such that there exists an $x$ with $|\operatorname{SP}(x)| \geq n$ satisfying $E$, and

$$
\tau_{n}^{(1 \mathrm{tt})}(E)=\sum_{N=1}^{\infty} \tau_{n}^{(\mathrm{lt})}(E ; N) \lambda^{-N}
$$

Finally, let

$$
\theta_{n}^{(1 \mathrm{t})}=\tau_{n}^{(\mathrm{lt})}(\Omega)
$$

where $\Omega$ is the whole probability space. Then we define

$$
\mathbb{Q}_{\infty}^{(1 \mathrm{tt})}(E)=\lim _{n \rightarrow \infty} \frac{\tau_{n}^{(1 \mathrm{lt})}(E)}{\theta_{n}^{(1 \mathrm{t})}}
$$

assuming the limit exists.

It should be possible to use the lace expansion to prove that the limit in (5.8) is well-defined, but this has not yet been done. The limit in (5.12) will be much more involved, since for this, one needs to understand the lattice tree survival probability $\theta_{n}^{(\mathrm{lt})}$. It is natural to conjecture that

$$
\rho_{\vec{m}}^{(1 \mathrm{t})}(\vec{x})=\mathbb{P}_{\infty}^{(\mathrm{lt})}\left(\left|\mathrm{SP}\left(x_{j}\right)\right|=m_{j} \forall j=1, \ldots, r-1\right)
$$

scales to the $r$-point function of ICSBM for $d>8$. That is the content of the next conjecture:

Conjecture 5.3. Let $d>8$. For all $r \geq 2, \vec{t}=\left(t_{1}, \ldots, t_{r-1}\right) \in \mathbb{R}^{r-1}$ and $\vec{k} \in \mathbb{R}^{d(r-1)}$, there exist constants $A, V, v$ and $\delta \in(0,1)$ such that (3.47) holds for $\rho^{(1 \mathrm{tt})}$.

\section{Conjectured scaling to ICSBM: Infinite structures}

So far, we have given a number of conjectures linking ICSBM to incipient infinite structures. We end this paper with two examples where the structures are infinite. 


\subsection{Invasion percolation above 6 dimensions}

We introduce the model for invasion percolation. For simplicity, we only define the model for a uniform step distribution $D$, such as the nearest-neighbour case or the case in (4.1). The bonds in these models are $\mathbb{B}=\{b=(u, v): D(u-v)>0\}$. We let $\{\omega(b)\}_{b \in \mathbb{B}}$ be a collection of i.i.d. uniform random variables. Given a random configuration $\omega$, we define a random increasing sequence of subgraphs $G_{0}, G_{1}, \ldots$ as follows. We let $G_{0}$ be the graph with no edges, and the single vertex 0 . We let $G_{i+1}=G_{i} \cup\left\{b_{i+1}\right\}$, where the edge $b_{i+1}$ is obtained by taking the $b \notin G_{i}$ with minimal $\omega(b)$ and such that $b$ has an end vertex in $G_{i}$. The invaded region is $\mathcal{S}=\cup_{i=0}^{\infty} G_{i}$. The law of the configuration of bonds in the invaded region is denoted by $\mathbb{P}^{(\mathrm{ip})}$ with corresponding expectation denoted by $\mathbb{E}^{(\mathrm{ip})}$.

It is well-known that the asymptotic behaviour of invasion percolation is closely related to the incipient cluster. The heuristic behind this is that $\limsup _{i \rightarrow \infty} \omega\left(b_{i}\right)=p_{c}$, which is the critical percolation threshold in the model [9]. In other words, asymptotically the invasion process only accepts values from critical clusters. As mentioned earlier, critical clusters in $d>6$ are fourdimensional, which leads to the well-known conjecture [54] that $\mathbb{P}(y$ is invaded $) \asymp|y|^{-(d-4)}$ when $d>6$. This conjecture is supported by results in [35, where the conjecture that

$$
\mathbb{P}(y \text { is invaded }) \asymp \mathbb{P}_{\infty}(0 \longrightarrow y) \asymp|y|^{-(d-4)}
$$

is explained in some detail. We can stretch this conjecture much further, and conjecture that the scaling limit of invasion percolation above 6 dimensions is ICSBM. For this, we define

$$
\rho_{\vec{n}}^{(\mathrm{ip})}(\vec{x})=\mathbb{P}^{(\mathrm{ip})}\left(\left|\operatorname{SP}\left(x_{j}\right)\right|=n_{j} \text { for each } j=1, \ldots, r-1\right),
$$

where now $|\mathrm{SP}(x)|$ is the minimal number of bonds in the invaded region along paths from 0 to $x$. Then we conjecture that $\rho_{\vec{n}}^{(\mathrm{ip})}$ converges to the moment measures of ICSBM:

Conjecture 6.1. Let $d>6$. For all $r \geq 2, \vec{t}=\left(t_{1}, \ldots, t_{r-1}\right) \in \mathbb{R}^{r-1}$ and $\vec{k} \in \mathbb{R}^{d(r-1)}$, there exist constants $A, V, v$ and $\delta \in(0,1)$ such that (3.47) holds for $\rho^{(\mathrm{ip})}$.

Conjecture 6.1 is quite hard to prove, as the relation between invasion percolation and unoriented percolation is not very direct. Maybe it would be simpler to investigate the following variant of invasion percolation, where we let $\{\omega(b)\}_{b \in \mathbb{B}}$ be a collection of i.i.d. uniform random variables on $\left[0, p_{c}\right]$ with probability $p_{c}$ and are equal to $\infty$ with probability $1-p_{c}$. In this case, instead of picking the smallest weight that is larger than $p_{c}$ when none below $p_{c}$ is available, we simply pick each of the boundary bonds of $G_{i}$ with equal probability.

\subsection{Uniform spanning forest above 4 dimensions}

The uniform spanning forest (USF) can be obtained as the weak limit of ordinary wired spanning trees on a large cube when the size of the cube tends to infinity. As it turns out, for $d \leq 4$, the USF consists of a single tree, while for $d>4$ it consists of multiple trees. See [3] and the references therein. In [3], there is a wealth of properties of USF's. For instance, a.s., the maximum over $x$ and $y$ of the number of edges outside the USF in a path from $x$ to $y$ equals $\left\lfloor\frac{d-1}{4}\right\rfloor$. Also, in [3], it is shown that the USF has stochastic dimension 4, which is a version of the statement that the trees that the USF consists of are four-dimensional.

Single uniform spanning trees, such as the spanning trees containing the origin, are natural candidates for convergence to ICSBM. To explain this in more detail, we need some notation. For $x \in \mathbb{Z}^{d}$, we let $T(x)$ be the (infinite) tree that contains $x$. Also, for $x \in T(y)$, we let $\operatorname{SP}(y, x)$ 
denote the path in the tree $T(x)=T(y)$ that goes from $x$ to $y$, and we let $\operatorname{SP}(y, x) \mid$ denote the number of bonds in this path and write $\mathrm{SP}(x)=\mathrm{SP}(0, x)$. Then, we define the $r$-point functions to be

$$
\rho_{\vec{n}}^{(\text {st) }}(\vec{x})=\mathbb{P}\left(x_{i} \in T(0),\left|\mathrm{SP}\left(x_{i}\right)\right|=n_{i} \forall i=1, \ldots, r-1\right) .
$$

"Wilson's method rooted at infinity" [64] can be used to generate the shortest path tree between any number of points using loop-erased random walk, and thus, allows us to give a probabilistic representation for the event that $x_{i} \in T(0)$ and $\left|\mathrm{SP}\left(x_{i}\right)\right|=n_{i}$ for all $i=1, \ldots, r-1$. Wilson's method rooted at infinity works on any transient graph. We start by generating an infinite simple random walk from the origin, and loop-erase it. Call the result $F_{1}$. Then, we start an simple random walk from $x_{1}$, and stop it when it hits $F_{1}$ (it is possible that it does not hit $F_{1}$ at all). After this, we loop-erase it, and call the union of the two loop-erased paths $F_{2}$. We can iterate this procedure. Denote by $F_{k-1}$ the union of the loop-eared paths from $0, x_{1}, \ldots, x_{k-1}$. Then we start a simple random walk from $x_{k}$ until it hits $F_{k-1}$, and subsequently loop-erase it, giving a self-avoiding path $\gamma_{k}$. Denote $F_{k}=F_{k-1} \cup \gamma_{k}$, and repeat the above procedure. To obtain the USF, we will have to go through all points of the graph $\mathbb{Z}^{d}$. However, the order in which the points are chosen is irrelevant, and thus, for the $r$-point function, it is convenient to start with $0, x_{1}, \ldots, x_{r-1}$. Clearly, when $x_{i} \in T(0)$ for all $i$, the result is the shortest path tree contained in $T(0)$ containing $x_{1}, \ldots, x_{r-1}$. Thus, $\rho_{\vec{n}}^{\text {(st) }}(\vec{x})$ equals the probability that $x_{i} \in T(0)$, and moreover, that the distance in $T(0)$ between 0 and $x_{i}$ equals $n_{i}$.

Wilson's construction shows that the behaviour of the USF is intimately related to loop-erased random walks (LERW). A lot is known about LERW, especially in dimensions $d \geq 4$. For $d>4$, LERW behaves diffusively, and the rescaled path converges to Brownian motion. See 49, Chapter 7] and the references therein. Therefore, we can think of the tree containing the origin and the points $x_{1}, \ldots, x_{r}$ as built up from an infinite path which scales to a Brownian motion (the LERW starting at the origin), and $r$-paths which also scale to Brownian motion and that are iteratively added to the infinite path. This picture of the $r$-point functions for the USF agrees with the picture of the $r$-point functions of ICSBM using the immortal particle. This leads us to the following conjecture:

Conjecture 6.2. Let $d>4$. For all $r \geq 2, \vec{t}=\left(t_{1}, \ldots, t_{r-1}\right) \in \mathbb{R}^{r-1}$ and $\vec{k} \in \mathbb{R}^{d(r-1)}$, there exist constants $A, V, v$ and $\delta \in(0,1)$ such that (3.47) holds for $\rho^{(\text {st) }}$.

\subsection{Discussion and notes for Sections 46}

We have presented a number of examples where the scaling limit is proven or conjectured to be ICSBM. Therefore, ICSBM is a natural and robust object that arises as a universal limit in a variety of models. We have focussed on convergence of the $r$-point functions to the moment measure of ICSBM. It would be of interest to prove stronger versions of convergence and to prove tightness. Tightness has proved to be difficult in all the models we have described in this paper.

The first results showing that spread-out oriented percolation above 4 spatial dimensions is Gaussian can be found in [55, 56], where the triangle condition is verified, and the two-point function is studied. The relation between oriented percolation and SBM in 39] was the greatest source of inspiration for this paper. Many more properties for oriented percolation, and its close brother, the contact process (see Section 5.2 below) are known. For example, in 60, hyperscaling inequalities are derived for these two models. From these hyperscaling inequalities, it follows that the mean-field critical exponents are restricted to $d \geq 4$. This identifies 4 as the upper critical 
dimension for oriented percolation. In [32], many related properties are proved for critical spreadout oriented percolation. For example, it is shown that the mass at time $m$, properly rescaled as in Theorem 3.7. converges to a size-biased exponential random variable, and that, conditioned to be alive at time $m, N_{m} / m$ weakly converges to an exponential random variable. Theorem 4.5 is not proved in [32, even though all the tools were available at that point.

The assumption in (4.8) is under investigation in [33, 34, where we investigate the critical survival probability, using the lace expansion.

For incipient infinite structures, we have a skeleton of a proof for the convergence towards ICSBM, by using convergence to SBM and Theorem 3.6. However, for infinite structures, this approach cannot be followed. Therefore, one would have to work with the $r$-point functions of the infinite structures directly, and prove convergence by investigating their scaling. It would be interesting, but probably quite difficult, to derive this scaling for one of the two examples.

For loop-erased random walk, it is known that also in the upper critical dimension, convergence towards Brownian motion holds, with logarithmic corrections. It would be of interest, but probably quite difficult, to extend this result to convergence towards ICSBM.

In [10, it is shown that the rescaled finite-range voter model converges to SBM for $d \geq 2$. Also, several related results, where local mean-field limits are taken, or for critical (continuous time) branching random walks, are considered. The proofs of these results are stronger than the ones for oriented percolation, since also tightness is proved. The reason that this problem is simpler is the fact that the dual process is coalescing random walks, and this is a simpler process. Thus, martingale methods can be used to prove the convergence to SBM. It would be of interest to investigate the link with the canonical measure further. Also, it can be expected that the voter model conditioned on non-extiction, and where we start with a single person having different opinion from all others, converges to ICSBM. Similar methods as in [10] are used in [17], where it was shown that the contact process converges to SBM when the range of the process grows with time. This mean-field limit with growing ranges makes the problem simpler than in the case where the range is large, but fixed, and this is the reason that SBM already appears as the limit when $d \geq 2$, rather than for $d>4$ as in Section 5.2

We close this discussion with an example of a tree in $\mathbb{Z}^{d}$ that is conjectured not to scale to ICSBM. For this, give each bond in $\mathbb{Z}^{d}$ a uniform weight in $[0,1]$. The minimal spanning forest (MSF) is the subgraph on $\mathbb{Z}^{d}$ where from each cycle we remove the edge with maximal weight. Then, it is conjectured that the stochastic dimension of the resulting structure equals 8 above 8 dimensions (see [3, Conjecture 6.7]. Since ICSBM is 4-dimensional, it cannot be expected that ICSBM can arise as the scaling limit of MSF. It would be of interest to (even heuristically) identify the scaling limit of MSF.

\section{Acknowledgements}

This work was supported in part by Netherlands Organisation for Scientific Research (NWO). I thank Gordon Slade for introducing me to the wonderful world of scaling limits and superprocesses, and for being able to explore this world together with him over the past years. Also, I thank Gordon for several other useful suggestions to improve the presentation in this paper. I also thank Takashi Hara, Frank den Hollander, Antal Járai and Akira Sakai, who have been my collaborators on research projects that have led to this article, and Frank Redig for stimulating discussions concerning the USF. I thank Mark Holmes and Akira Sakai for useful remarks on early versions of this paper. Finally, I thank Achim Klenke, Jean-François Le Gall and Ed Perkins for 
clarifying some of the subtleties of super-processes.

\section{References}

[1] M. Aizenman. On the number of incipient spanning clusters. Nucl. Phys. B [FS], 485:551-582, (1997).

[2] D. Aldous. The continuum random tree III. Ann. Probab., 21:248-289, (1993).

[3] I. Benjamini, H. Kesten, Y. Peres and O. Schramm. Geometry of the Uniform Spanning Forest: Transitions in Dimensions 4, 8, 12, . . Preprint, arXiv:math.PR/0107140, (2002). To appear in Ann. Math..

[4] I. Benjamini, R. Lyons, Y. Peres and O. Schramm. Uniform spanning forests. Ann. Probab., 29:1-65, (2001).

[5] C. Bezuidenhout and G. Grimmett. The critical contact process dies out. Ann. Probab., 18:1462-1482, (1990).

[6] C. Bezuidenhout and G. Grimmett. Exponential decay for subcritical contact and percolation processes. Ann. Probab. 19 (1991): 984-1009.

[7] P. Billingsley. Probability and Measure. John Wiley and Sons, New York, 3rd edition, (1995).

[8] C. Borgs, J. Chayes, R. van der Hofstad and G. Slade. Mean-field lattice trees. Ann. Combinatorics, 3: 205-221 (1999).

[9] J.T. Chayes, L. Chayes and C.M. Newman. Stochastic geometry of invasion percolation. Commun. Math. Phys., 101 383-407, (1985).

[10] J.T. Cox, R. Durrett, and E.A. Perkins. Rescaled voter models converge to super-Brownian motion. Ann. Probab., 28:185-234, (2000).

[11] J.T. Cox, R. Durrett, and E.A. Perkins. Rescaled particle systems converging to superBrownian motion. In M. Bramson and R. Durrett, editors, Perplexing Problems in Probability: Festschrift in Honor of Harry Kesten, Basel, (1999). Birkhäuser.

[12] D.A. Dawson. Measure-valued Markov processes. In Ecole d'Eté de Probabilités de SaintFlour 1991. Lecture Notes in Mathematics \#1541, Berlin, (1993). Springer.

[13] D.A. Dawson, I. Iscoe and E.A. Perkins. Super-Brownian motion: path properties and hitting probabilities. Probab. Th. Rel. Fields, 83:135-205, (1989).

[14] D.A. Dawson and E.A. Perkins. Measure-valued processes and renormalization of branching particle systems. Stochastic partial differential equations: six perspectives, Math. Surveys Monogr. 64: 45-106, Amer. Math. Soc., Providence, RI, (1999).

[15] E. Derbez and G. Slade. Lattice trees and super-Brownian motion. Canad. Math. Bull., 40:19-38, (1997).

[16] E. Derbez and G. Slade. The scaling limit of lattice trees in high dimensions. Commun. Math. Phys., 193:69-104, (1998). 
[17] R. Durrett and E.A. Perkins. Rescaled contact processes converge to super-Brownian motion in two or more dimensions. Probab. Th. Rel. Fields, 114:309-399, (1999).

[18] E.B. Dynkin. Representation for functionals of superprocesses by multiple stochastic integrals, with applications to self-intersection local times. Astérisque, 157-158:147-171, (1988).

[19] E.B. Dynkin. An Introduction to Branching Measure-Valued Processes. American Mathematical Society, Providence, (1994).

[20] N. El Karoui and S. Roelly. Martingale properties, explosion and Levy-Khinchin representation of a class of measure-valued branching processes. (French) Stochastic Process. Appl., 38(2): 239-266, (1991).

[21] A.M. Etheridge. An Introduction to Superprocesses. American Mathematical Society, Providence, (2000).

[22] S.N. Evans. Two representations of conditioned superprocesses. Proc. Roy. Soc. Edin. Ser. A, 123 part 5, 959-971 (1993).

[23] S.N. Evans and E. Perkins. Measure-valued Markov branching processes conditioned on nonextinction. Israel J. Math., 71 no. 3, 329-337 (1990).

[24] J. Geiger. Elementary new proofs of classical limit theorems for Galton-Watson processes. J. Appl. Probab., 36 no. 2, 301-309 (1999).

[25] G. Grimmett. Percolation. Springer, Berlin, 2nd edition, (1999).

[26] G. Grimmett and P. Hiemer. Directed percolation and random walk. In: V. Sidoravicius, editor, In and Out of Equilibrium, pages 273-297. Birkhäuser, Boston, (2002).

[27] H. Haase. On the incipient cluster of the binary tree. Arch. Math. (Basel), 63: no. 5, 465-471 (1994).

[28] T. Hara, R. van der Hofstad and G. Slade. Critical two-point functions and the lace expansion for spread-out high-dimensional percolation and related models. Ann. Probab. 31 349-408, (2003).

[29] T. Hara and G. Slade. The scaling limit of the incipient infinite cluster in high-dimensional percolation. I. Critical exponents. J. Statist. Phys., 99 1075-1168, (2000).

[30] T. Hara and G. Slade. The scaling limit of the incipient infinite cluster in high-dimensional percolation. II. Integrated super-Brownian excursion. J. Math. Phys., 41:1244-1293, (2000).

[31] R. van der Hofstad, F. den Hollander and G. Slade. A new inductive approach to the lace expansion for self-avoiding walks. Probab. Th. Rel. Fields 111 (1998): 253-286.

[32] R. van der Hofstad, F. den Hollander, and G. Slade. Construction of the incipient infinite cluster for spread-out oriented percolation above $4+1$ dimensions. Commun. Math. Phys, 231: 435-461 (2002).

[33] R. van der Hofstad, F. den Hollander, and G. Slade. The survival probability for spread-out oriented percolation above $4+1$ dimensions. I: Induction. In preparation. 
[34] R. van der Hofstad, F. den Hollander, and G. Slade. The survival probability for spread-out oriented percolation above $4+1$ dimensions. II: Expansion. In preparation.

[35] R. van der Hofstad and A. Járai. The incipient infinite cluster for high-dimensional unoriented percolation. Journal of Stat. Phys., 114:625-663 (2004).

[36] R. van der Hofstad and A. Sakai. Gaussian scaling for the critical spread-out contact process above the upper critical dimension. Preprint 2003.

[37] R. van der Hofstad and A. Sakai. Convergence of the critical finite-range contact process to super-Brownian motion above the upper critical dimension. In preparation.

[38] R. van der Hofstad and G. Slade. A generalised inductive approach to the lace expansion. Probab. Th. Rel. Fields, 122:389-430, (2002).

[39] R. van der Hofstad and G. Slade. Convergence of critical oriented percolation to superBrownian motion above $4+1$ dimensions. Ann. Inst. Henri Poincaré, 39: 413-485 (2003).

[40] M. Holmes. Long-lived lattice trees above 8 dimensions. PhD thesis, in preparation.

[41] A. Járai Jr. Incipient infinite percolation clusters in 2d. Ann. Probab., 31:444-485, (2003).

[42] A. Járai Jr. Invasion percolation and the incipient infinite cluster in 2d. Commun. Math. Phys, 236: 311-334, (2003).

[43] O. Kallenberg. Random measures. Third edition. Akademie-Verlag, Berlin. Academic Press, Inc., (1983).

[44] H. Kesten. The incipient infinite cluster in two-dimensional percolation. Probab. Th. Rel. Fields, 73:369-394, (1986).

[45] H. Kesten. Subdiffusive behavior of random walk on a random cluster. Ann. Inst. Henri Poincaré, 22: 425-487, (1986).

[46] H. Kesten. Branching random walk with a critical branching part. J. Theoret. Probab., 8: , no. 4, 921-962, (1995).

[47] A.N. Kolmogorov. Zur Lösung einer biologoschen Aufgabe. Izv. NII Mathem. Mekh. Tomskogo Univ. 2:1-6, (1938).

[48] J. Lamperti and P. Ney. Conditioned branching processes and their limiting diffusions. Theory Probab. Appl. 13: 126-137, (1968).

[49] G.F. Lawler. Intersections of random walks. Birkhäuser, Basel, (1991).

[50] J.-F. Le Gall. Spatial Branching Processes, Random Snakes, and Partial Differential Equations. Birkhäuser, Basel, (1999).

[51] Z.H. Li and T. Shiga. Measure-valued branching diffusions: immigrations, excursions and limit theorems. J. Math. Kyoto Univ., 35(2):233-274, (1995).

[52] T. Liggett. Stochastic Interacting Systems: Contact, Voter and Exclusion Processes. Springer, Berlin (1999). 
[53] R. Lyons, R. Pemantle and Y. Peres. Conceptual proofs of $L \log L$ criteria for mean behaviour of branching processes. Ann. Probab., 25:1125-1138, (1995).

[54] C. M. Newman and D. L. Stein. Ground-state structure in a highly disordered spin-glass model. J. Statist. Phys. 82 1113-1132, (1996).

[55] B.G. Nguyen and W-S. Yang. Triangle condition for oriented percolation in high dimensions. Ann. Probab., 21:1809-1844, (1993).

[56] B.G. Nguyen and W-S. Yang. Gaussian limit for critical oriented percolation in high dimensions. J. Stat. Phys., 78:841-876, (1995).

[57] E. Perkins. Dawson-Watanabe superprocesses and measure-valued diffusions. In P.L. Bernard, editor, Lectures on Probability Theory and Statistics. Ecole d'Eté de Probabilités de SaintFlour XXIX-1999, pages 125-329, Berlin, (2002). Springer. Lecture Notes in Mathematics $\# 1781$.

[58] R. Rammal and G. Toulouse. Random walk on fractal structures and percolation clusters. J. Physique Lett. 44 L13-L22, (1983).

[59] A. Sakai. Mean-field critical behavior for the contact process. J. Statist. Phys. 104: 111-143, (2001).

[60] A. Sakai. Hyperscaling inequalities for the contact process and oriented percolation, J. Stat. Phys. 106: 201-211 (2002).

[61] T. Salisbury and J. Verzani. Non-degenerate conditionings of the exit measures of super Brownian motion. Stoch. Processes and Applic. 87: 25-52, (2000).

[62] B. Simon. Functional Integration and Quantum Physics. Academic Press, New York, (1979).

[63] G. Slade. Scaling limits and super-Brownian motion. Notices A.M.S., 49(9):1056-1067, (2002).

[64] D. Wilson. Generating random trees more quickly than the cover time. Proceedings of the 28th Annual ACM Symposium on the Theory of Computing (Philadelphia, PA, 1996), 296-303, ACM, New York, (1996).

[65] A.M. Yaglom. Certain limit theorems of the theory of branching random processes. (Russian) Doklady Akad. Nauk SSSR (N.S.) 56: 795-798, (1947). 